\title{
Quantitative study on helicity inversion in Majorana neutrino decays at the LHC
}

\author{
Richard Ruiz@" \\ Centre for Cosmology, Particle Physics and Phenomenology (CP3), Université Catholique de Louvain, \\ Chemin du Cyclotron, Louvain la Neuve B-1348, Belgium \\ and Institute of Nuclear Physics, Polish Academy of Sciences, ul. Radzikowskiego, Cracow 31-342, Poland
}

(Received 7 August 2020; accepted 6 January 2021; published 20 January 2021)

\begin{abstract}
We report an analytical and numerical investigation into the impact of helicity inversion in LHC processes that do not conserve lepton number $(L)$. As a case study, we focus on the production and decay of Majorana neutrinos $(N)$ through on- and off-shell $W$ bosons in the phenomenological type I seesaw model. Using the Monte Carlo event generator MadGraph5_aMC@NLO in conjunction with the HeavyN model libraries, we perform exact matrix element (ME) computations without the narrow width approximation. Despite helicity inversion appearing explicitly in MEs, we report the absence of helicity suppression of $L$-violating collider observables for $1 \rightarrow 4$ and $2 \rightarrow 4$ processes that are dominated by resonant $N$ production. We attribute this incongruity to the different scalings of 4-momenta and squared 4-momenta in MEs and squared MEs, with exact cancelations occurring in the latter when $N$ goes onshell in the small-width limit. In off-shell regimes, total suppression/enhancement of $L$ violation can emerge. Implications for other neutrino mass models are discussed.
\end{abstract}

DOI: $10.1103 /$ PhysRevD.103.015022

\section{INTRODUCTION}

Among the outstanding questions in particle physics [1] is whether the light neutrinos observed in nature $(\nu)$ are their own antiparticle, i.e., are they Majorana fermions? If so, then the Lagrangian of the Standard Model of particle physics (SM), which stipulates that neutrinos are massless, must be extended by dimensionful operators that violate the SM's conservation of lepton number $(L)$. Gauge invariance and renormalizability, however, require that such operators have ultraviolet completions, and thereby suggests the possibility of new particles [2]. Hence, discovering the Majorana nature of neutrinos may be a stepping-stone to realizing a mechanism for neutrino massgeneration [3-16], new gauge forces [7,13,17-20], or even grand unification $[5,8,12,15,21,22]$.

Despite this importance, however, direct tests of neutrinos' Majorana nature, such as through searches for neutrinos' magnetic dipole moments or through $|\Delta L|=$ 2 transitions like neutrinoless $\beta \beta$ decay $(0 \nu \beta \beta)$, are encumbered by manifestations of the so-called DiracMajorana confusion theorem [23,24]. In the absence of

richard.ruiz@uclouvain.be

Published by the American Physical Society under the terms of the Creative Commons Attribution 4.0 International license. Further distribution of this work must maintain attribution to the author(s) and the published article's title, journal citation, and DOI. Funded by SCOAP ${ }^{3}$. new particles, the theorem in its standard formulation [24] shows that an inherent helicity inversion in such processes leads to matrix elements (ME) being proportional to light neutrino masses. This implies that transition probabilities formally vanish in the limit of vanishing neutrino masses, and thus are classified as being helicity suppressed. As such, two complementary approaches to the "Majorana" question are embraced: The first is the development of large-scale facilities that, for example, aim to measure the $0 \nu \beta \beta$ decay rate. The second relies on direct searches for $|\Delta L|=2$ processes in the context of neutrino mass models. For reviews of these approaches, see Refs. [25-30].

In the second approach, processes that do not conserve $L$ are mediated by new particles [3-16] that are typically much heavier than light neutrinos, but possibly lighter than the electroweak (EW) scale. Crucially, the confusion theorem follows from rather generic kinematical arguments in the context of chiral gauge theories, e.g., the EW theory, and not on flavor symmetries as considered, for example, in Refs. [31-34]. As such, in scenarios with heavy Majorana neutrinos $(N)$, helicity inversion manifests as asymmetries in angular distributions that distinguish $|\Delta L|=0$ and $|\Delta L|=2$ channels [35-43]. However, while generalizations of the theorem show [35] that these MEs are consistently proportional to heavy neutrino masses $\left(m_{N}\right)$, past studies have not specifically investigated whether the MEs also vanish when $m_{N}$ do. For resonantly produced Majorana neutrinos this is pertinent as the often-quoted equality of $|\Delta L|=0$ and $|\Delta L|=2$ decay rates, which 


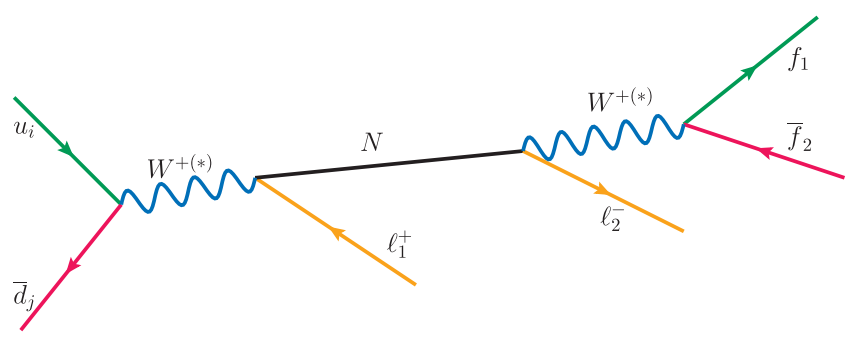

(a)

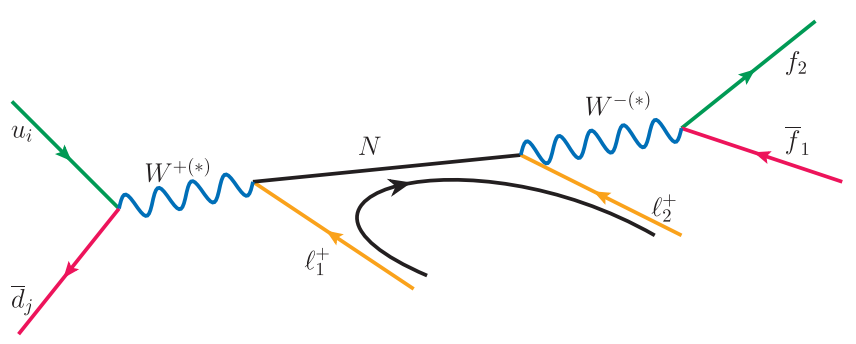

(b)

FIG. 1. Born-level, diagrammatic representation of the (a) $L$-conserving process $u \bar{d} \rightarrow W^{+} \rightarrow N \ell_{1}^{+} \rightarrow \ell_{1}^{+} \ell_{2}^{-} f_{1} \bar{f}_{2}$, and (b) its $L$-violating analogue $u \bar{d} \rightarrow W^{+} \rightarrow N \ell_{1}^{+} \rightarrow \ell_{1}^{+} \ell_{2}^{+} \bar{f}_{1} f_{2}$. Interfering diagrams not shown. Drawn with Jaxodraw [51].

implies the absence of helicity-suppression, assumes the narrow width approximation and that resonant $N$ can be treated as unpolarized states $[44,45]$. This is despite the presence of chiral couplings and that needed criteria may not be satisfied for currents with Majorana fermions [46-50].

In this study, we report an analytical and numerical investigation into the impact of helicity inversion in $L$-violating transition rates involving heavy Majorana neutrinos at the $\sqrt{s}=13 \mathrm{TeV}$ Large Hadron Collider (LHC). As a representative case study, we work in the framework of the phenomenological type I seesaw model and focus on the $L$-violating decay and scattering processes [44]

$$
\begin{gathered}
W^{ \pm} \rightarrow e_{1}^{ \pm} N^{(*)} \rightarrow e_{1}^{ \pm} e_{2}^{ \pm} j j, \\
p p \rightarrow W^{ \pm(*)} \rightarrow e_{1}^{ \pm} N^{(*)} \rightarrow e_{1}^{ \pm} e_{2}^{ \pm} j j,
\end{gathered}
$$

and their $L$-conserving counterparts, as shown diagrammatically at the parton level in Fig. 1. While Eqs. (1.1) and (1.2) are intimately related, their individual considerations explore subtle polarization and virtuality effects.

By performing exact $\mathrm{ME}$ computations with the Monte Carlo (MC) event generator MadGraph5_aMC@NLO $[52,53]$ in conjunction with the HeavyN model libraries $[54,55]$, and without invoking the narrow width approximation, we find that the helicity suppression in collider observables is numerically negligible for processes driven by resonant production of Majorana neutrinos with masses $m_{N}>1 \mathrm{GeV}$ and total widths $\Gamma_{N} \ll m_{N}$. We attribute the seeming incongruity with the presence of helicity inversion to the different scaling of 4-momenta and squared 4-momenta in MEs and squared MEs. In the on-shell, small-width limit, this leads to cancelations of the dependence on $m_{N}$, with corrections proportional to off-shell virtualities and $\Gamma_{N}$. Outside this limit we observe the opposite behavior. When the off-shell behavior is driven by a large width, we find that the $|\Delta L|=2$ channel is helicity-suppressed; when the off-shell behavior is driven by a too large mass, helicity enhancement emerges. As the arguments here are kinematical in nature, analogous findings apply to other models with Majorana $N$.

This study continues in the following order: In Sec. II we summarize the theoretical framework in which we work. In Sec. III we document our computational setup. We then identify analytically in Sec. IV the helicity inversion at the ME level, its propagation to the squared ME level, and its (mis)cancelation in the (off)on-shell limit for the processes in Fig. 1. We also comment on implications for other models and $|\Delta L|=2$ processes. We present our numerical comparisons in Sec. V and conclude in Sec. VI.

\section{THEORETICAL FRAMEWORK}

To investigate the potential helicity suppression of $L$-violating processes mediated by Majorana neutrinos, we work in the framework of the phenomenological type I seesaw. In this well-documented [25,56,57] scenario, the masses and mixing angles of light $\left(\nu_{k}\right)$ and heavy $\left(N_{k^{\prime}}\right)$ neutrino mass eigenstates are decoupled in order to conduct flavor model-independent studies and searches.

In this model, the SM's field content is extended by $n_{R} \geq 3$ right-handed $(\mathrm{RH})$ neutrinos $\left(\nu_{R}^{i}\right)$ that are gaugesinglets, i.e., are chargeless/sterile, under the SM gauge interactions. This allows the $\nu_{R}^{i}$ to possess RH Majorana masses $\left(\mu_{R}^{i j}\right)$, which violate $L$ conservation and can, in principle, can acquire any value. ${ }^{1}$ The decoupling of $\mu_{R}^{i j}$ subsequently suppresses light neutrino masses [3,5-10] and is distinct from other neutrino mass mechanisms, e.g., the type II seesaw [4,10-12], where $\nu$ masses are generated via left-handed (LH) Majorana masses.

Accordingly, the Lagrangian of the phenomenological type I seesaw $\left(\mathcal{L}_{\text {Type I }}\right)$ is characterized by extending the SM Lagrangian $\left(\mathcal{L}_{\mathrm{SM}}\right)$ at the renormalizable level by kinetic and mass terms for the $\nu_{R}^{i}\left(\mathcal{L}_{\mathrm{Kin}}\right)$, and by Yukawa couplings $\left(\mathcal{L}_{\mathrm{Y}}\right)$ between the $\nu_{R}^{i}$, the SM Higgs field $(\Phi)$, and the SM's LH lepton doublets $L^{j T}=\left(\nu_{L}^{j}, l_{L}^{j}\right)$,

\footnotetext{
${ }^{1}$ If coupled to other physics, e.g., particle dark matter [58,59] or global symmetries [31-34,60-62], then the values of $\mu_{R}^{i j}$ can be stringently constrained.
} 


$$
\mathcal{L}_{\text {Type I }}=\mathcal{L}_{\mathrm{SM}}+\mathcal{L}_{\text {Kin }}+\mathcal{L}_{\mathrm{Y}}
$$

After EWSB and diagonalizing charged lepton flavor states into their mass eigenstates $(\ell=e, \mu, \tau)$, the flavor eigenstates of active, LH neutrinos $\left(\nu_{L \ell}\right)$ can be decomposed into mass eigenstates via the rotation [25]

$$
\nu_{L \ell}=\sum_{k=1}^{3} U_{\ell k} \nu_{k}+\sum_{k^{\prime}=1}^{n_{R}} V_{\ell k^{\prime}} N_{k^{\prime}} .
$$

Here the complex-valued mixing elements $U_{\ell k}$ and $V_{\ell k^{\prime}}$ parametrize the mixing between the flavor state $\nu_{L \ell}$ with the mass eigenstates $\nu_{k}$ and $N_{k^{\prime}}$. For updated measurements and constraints of mixing angles, see Refs. [63-66].

Given Eq. (2.2), the relevant interaction Lagrangian describing the charged current interactions of $N_{k^{\prime}}$ is,

$$
\begin{aligned}
\mathcal{L}= & -\frac{g_{W}}{\sqrt{2}} W_{\mu}^{+} \sum_{\ell}^{\tau}\left[\overline{\nu_{L \ell}} \gamma^{\mu} P_{L} \ell\right]+\text { H.c. } \\
= & -\frac{g_{W}}{\sqrt{2}} W_{\mu}^{+} \sum_{k=1}^{3} \sum_{\ell}^{\tau}\left[\overline{\nu_{k}} U_{\ell k}^{*} \gamma^{\mu} P_{L} \ell\right] \\
& -\frac{g_{W}}{\sqrt{2}} W_{\mu}^{+} \sum_{k^{\prime}=1}^{n_{R}} \sum_{\ell}^{\tau}\left[\overline{N_{k^{\prime}}} V_{\ell k^{\prime}}^{*} \gamma^{\mu} P_{L} \ell\right]+\text { H.c. }
\end{aligned}
$$

Here, $g_{W}=e / \sin \theta_{W} \approx 0.65$ is the usual weak gauge coupling constant in the SM, and $P_{L / R}=(1 / 2)\left(1 \mp \gamma^{5}\right)$ are the $\mathrm{LH} / \mathrm{RH}$ chiral projection operators in fourcomponent notation. Using Eq. (2.2) to make analogous substitutions, interaction Lagrangians involving the $Z$ and Higgs can be built accordingly [25,56]. Throughout this study we consider the impact of only the lightest heavy mass eigenstate $\left(N_{k^{\prime}=1}\right)$, which we relabel as $N \equiv N_{k^{\prime}=1}$ with $V_{\ell N} \equiv V_{\ell k^{\prime}=1}$. We do so to isolate the impact of helicity inversion in $L$-violating currents that can otherwise be obfuscated by strong interference.

\section{COMPUTATIONAL SETUP}

We now briefly document the computational setup of this study. After summarizing the MC setup in Sec. III A, the numerical inputs for SM and heavy neutrino parameters are respectively provided in Secs. III B and III C.

\section{A. Monte Carlo setup}

To perform our numerical computations, we use the $\mathrm{MC}$ event generator MadGraph5_aMC@NLO (v2.7.0) [52,53] (MGAMC). The simulation suite [50,52,53,67-70] operates by constructing helicity amplitudes for short-distance decay and scattering processes $[52,68,71]$ according formalism of Refs. [71-74] and performs fast numerical integration over phase space through MC sampling $[67,75]$. For heavy neutrino interactions governed by the
Lagrangian of Eq. (2.4), we import into MGAMC the HeavyN [54,55] FeynRules [76-78] libraries. This employs the conventions for Majorana currents developed in Refs. [79,80]. For select calculations, we compute helicity-polarized MEs in MGAMC according to the formalism of Ref. [81].

\section{B. Standard model inputs}

For numerical computations we work in the $n_{f}=5$ massless/active quarks scheme with SM inputs set to

$$
\begin{aligned}
& m_{t}\left(m_{t}\right)=173.3 \mathrm{GeV}, \quad M_{Z}=91.1876 \mathrm{GeV}, \\
& \alpha_{\mathrm{QED}}^{-1}\left(M_{Z}\right)=127.94, \quad G_{F}=1.174560 \times 10^{-5} \mathrm{GeV}^{-2} .
\end{aligned}
$$

We take the Cabbibo-Kobayashi-Maskawa matrix equal to the identity matrix. For relevant computations we use the MSTW 2008 leading order parton density functions (1haid=21000) [82] as evolved by LHAPDF (v6.2.3) [83], and set the collinear factorization scale $\left(\mu_{f}\right)$ to

$$
\mu_{f}=M_{W} \approx 79.95 \mathrm{GeV} .
$$

\section{Heavy neutrino inputs}

In addition to SM inputs, the (default) HeavyN model libraries [54,55] consists of three Majorana neutrino mass eigenstates $N_{k^{\prime}}$ with mass eigenvalues $m_{N_{k^{\prime}}}$ and activesterile mixing elements $V_{\ell k^{\prime}}$ associated with lepton flavor $\ell$. As explained at the end of Sec. II, we decouple two $N_{k}$ in order to isolate helicity inversion in the absence of interference. To do this numerically, we set

$m_{N_{2}}, m_{N_{3}}=10^{12} \mathrm{GeV}$ and $\left|V_{\ell 2}\right|,\left|V_{\ell 3}\right|=10^{-10}$.

As the values of $m_{N_{1}},\left|V_{\ell N_{1}}\right|$ are varied, the total width $\left(\Gamma_{N_{1}}\right)$ of $N_{1}$ is evaluated ${ }^{2}$ on-the-fly using MadDecay [70].

\section{HELICITY INVERSION IN MATRIX ELEMENTS WITH MAJORANA NEUTRINOS}

For $|\Delta L|=2$ transitions that are mediated by heavy Majorana neutrinos in the phenomenological Type I seesaw, we establish in this section the presence of helicity

\footnotetext{
${ }^{2}$ We note that there is a limited ability in MadDecay to compute extremely small particle widths, which can occur for particularly tiny mixing elements. In this study, no such threshold was reached. However, a possible workaround for future studies would be to evaluate a total width at an artificially large mixing element and rescale to much smaller ones. For example: one can compute $\Gamma_{N}\left(\left|V_{\ell N}\right|=10^{-3}\right)=\left(10^{-3} / 10\right)^{2} \times \Gamma_{N}\left(\left|V_{\ell N}\right|=10\right)$.
} 
inversion in MEs and its propagation into squared MEs. While the inversion has far-reaching consequences $[23,24,35-43]$, it is essentially a quirk of chiral gauge theories, like the EW theory, and follows from the chargeparity-time $(C P T)$ theorem. We organize this derivation by first considering $L$-conserving, 4-body decays of $W$ bosons in Secs. IVA, and then $L$-violating decays in Secs. IV B. We draw special attention to the precise origin of the inversion and the scaling of (squared) momenta in (squared) MEs. In Sec. IV D we consider analogous processes in $2 \rightarrow 4$ scattering and comment on the implications for other neutrino mass models in Sec. IV E.

\section{A. $W$ boson decays with $\Delta L=0$}

As a first step to studying helicity inversion in $|\Delta L|=2$ processes, we consider the following $L$-conserving, 4-body $W$ boson decay mediated by a Majorana neutrino $N$,

$$
\begin{aligned}
W_{\lambda_{W}}^{+}\left(p_{W}\right) & \rightarrow \ell_{R 1}^{+}\left(p_{1}\right) N_{\lambda_{N}}\left(p_{N}\right) \\
& \rightarrow \ell_{R 1}^{+}\left(p_{1}\right) \ell_{L 2}^{-}\left(p_{2}\right) c_{L}\left(p_{c}\right) \overline{s_{R}}\left(p_{s}\right),
\end{aligned}
$$

as shown as a subprocess in Fig. 1(a). Here, the subscripts $\lambda_{W}=0, \pm 1$ and $\lambda_{N}=L, R$ denote the helicities of $W^{+}$and $N$. The helicities of massless fermions $\ell_{k}, c, s$ are fixed by the $W$ boson's chiral couplings.

Working in the unitary gauge and in the HELAS basis [71] for helicity amplitudes, the corresponding ME is

$$
\begin{aligned}
-i \mathcal{M}_{L}^{W}= & \varepsilon_{\mu}\left(p_{W}, \lambda_{W}\right) T_{L}^{\nu \mu}\left(p_{1}, p_{2}, p_{N}\right) \\
& \times \Delta_{\nu \rho}\left(p_{c}+p_{s}\right) J^{\rho}\left(p_{c}, p_{s}\right) .
\end{aligned}
$$

Here the $(c \bar{s})$ fermion current and $W$ propagator are

$$
\begin{gathered}
J^{\rho}\left(p_{c}, p_{s}\right)=\frac{-i g_{W} \delta^{A B}}{\sqrt{2}}\left[\bar{u}_{L}^{A}\left(p_{c}\right) \gamma^{\rho} P_{L} v_{R}^{B}\left(p_{s}\right)\right], \\
\Delta_{\nu \rho}(k)=\frac{-i\left(g_{\nu \rho}-k_{\nu} k_{\rho} / M_{W}^{2}\right)}{\left(k^{2}-M_{W}^{2}+i \Gamma_{W} M_{W}\right)},
\end{gathered}
$$

and the $L$-conserving $\left(\ell_{1}^{+} N \ell_{2}^{-}\right)$lepton current is

$$
\begin{gathered}
T_{L}^{\nu \mu}\left(p_{1}, p_{2}, p_{N}\right)=\left(\frac{-i g_{W}}{\sqrt{2}}\right)^{2} V_{\ell_{1} N}^{*} V_{\ell_{2} N} \times \mathcal{N}_{L}^{\nu \mu} \times \mathcal{D}, \\
\mathcal{N}_{L}^{\nu \mu}=\left[\bar{u}_{L}\left(p_{2}\right) \gamma^{\nu} P_{L}\left(\not p_{N}+m_{N} \mathbb{I}_{4}\right) \gamma^{\mu} P_{L} v_{R}\left(p_{1}\right)\right], \\
\mathcal{D}=\frac{i}{\left(p_{N}^{2}-m_{N}^{2}+i \Gamma_{N} m_{N}\right)} .
\end{gathered}
$$

In the quark current $J^{\rho}$, the indices $A, B=1, \ldots, N_{c}=3$ run over the QCD color states, and the Kronecker $\delta$-function $\delta^{A B}$ ensures a color-singlet $W^{*} \rightarrow q \bar{q} \bar{q}^{\prime}$ splitting. In the lepton current $T_{L}^{\nu \mu}, \mathcal{D}$ is the pole structure of the Breit-Wigner propagator for the Majorana neutrino $N$.

Importantly, the $\left(\ell_{1}^{+} N \ell_{2}^{-}\right)$fermion current is initiated/ terminated by successive $W$ interactions. These are maximally parity-violating, are oriented in the left chiral direction, and are responsible for the two $\left(\gamma^{\alpha} P_{L}\right)$ in $\mathcal{N}_{L}^{\nu \mu}$. Due to orthogonality of RH and LH chiral projection operators, the intermediate $N$ is confined to its LH helicity state (the $\not \not_{N}$ term). The transition is helicity conserving as RH helicity states (the $m_{N} \mathbb{I}_{4}$ term) do not contribute to successive chiral interactions with the same chirality.

After anticommuting the left-most $P_{L}$ and using naïve power counting to extract the energy dependence from spinors, we obtain for the $\left(\ell_{1}^{+} N \ell_{2}^{-}\right)$lepton current:

$$
\begin{aligned}
\mathcal{N}_{L}^{\nu \mu} & =\left[\bar{u}_{L}\left(p_{2}\right) \gamma^{\nu} P_{L}\left(\not \not{ }_{N}+m_{N} \mathbb{I}_{4}\right) \gamma^{\mu} P_{L} v_{R}\left(p_{1}\right)\right] \\
& =\left[\bar{u}_{L}\left(p_{2}\right) \gamma^{\nu} \not \not_{N} \gamma^{\mu} P_{L} v_{R}\left(p_{1}\right)\right] \\
& \sim \sqrt{E_{2}} E_{N} \sqrt{E_{1}} \sim M_{W}^{2} .
\end{aligned}
$$

The scaling in the last line shows that the amplitude $\mathcal{M}_{L}^{W}$ for the $W^{+} \rightarrow \ell_{1}^{+} \ell_{2}^{-} c \bar{s}$ decay grows with the energy of $N$, and therefore is not suppressed for vanishing $m_{N}$.

We note that due to Lorentz invariance the scaling of 4-momenta $\left(p^{\mu}\right)$ and their squares $\left(p^{\mu} p_{\mu}\right)$ differ. Importantly, this leads to different behavior in squared MEs than in Eq. (4.11). In particular, one finds using Ref. [35],

$$
\begin{aligned}
\sum\left|\mathcal{M}_{L}^{W}\right|^{2} & \sim \sum T_{L}^{\nu \mu}\left[T_{L}^{\alpha \beta}\right]^{\dagger} \\
& \sim \sum \mathcal{N}_{L}^{\nu \mu}\left[\mathcal{N}_{L}^{\alpha \beta}\right]^{\dagger} \times\left|\mathcal{D}\left(p_{N}^{2}\right)\right|^{2},
\end{aligned}
$$

where the squared and spin-summed current scales as

$$
\sum \mathcal{N}_{L}^{\nu \mu}\left[\mathcal{N}_{L}^{\alpha \beta}\right]^{\dagger} \sim E_{2} p_{N}^{2} E_{1} \sim M_{W}^{2} p_{N}^{2} .
$$

Interestingly, Eq. (4.14) shows that the squared ME scales as the virtuality of the intermediate $N$, and can potentially vanish for tiny $m_{N}$ in the on-shell limit. In this region of phase space however, i.e., when

$$
\delta p_{N}^{2} \equiv\left|p_{N}^{2}-m_{N}^{2}\right| \ll \Gamma_{N} m_{N} \ll m_{N}^{2},
$$

the pole structure of the propagator $\mathcal{D}$ behaves as

$$
\begin{aligned}
\left|\mathcal{D}\left(p_{N}^{2}\right)\right|^{2} & =\frac{1}{\left(p_{N}^{2}-m_{N}^{2}\right)^{2}+\left(\Gamma_{N} m_{N}\right)^{2}} \\
& =\frac{1}{\left(\Gamma_{N} m_{N}\right)^{2}\left[1+\frac{\delta p_{N}^{4}}{\left(\Gamma_{N} m_{N}\right)^{2}}\right]}
\end{aligned}
$$




$$
=\frac{1}{\left(\Gamma_{N} m_{N}\right)^{2}}\left[1-\mathcal{O}\left(\frac{\delta p_{N}^{4}}{\left(\Gamma_{N} m_{N}\right)^{2}}\right)\right] .
$$

In combination with the scaling in Eq. (4.14), we obtain ${ }^{3}$

$$
\sum\left|\mathcal{M}_{L}^{W}\right|^{2} \sim \frac{M_{W}^{2} p_{N}^{2}}{\left(\Gamma_{N} m_{N}\right)^{2}}\left[1-\mathcal{O}\left(\frac{\delta p_{N}^{4}}{\left(\Gamma_{N} m_{N}\right)^{2}}\right)\right],
$$

and see that the dependence on $m_{N}^{2}$ is actually cancelled in the on-shell limit. Hence, like at the ME level, the leading contribution to the $W^{+} \rightarrow \ell_{1}^{+} \ell_{2}^{-} c \bar{s}$ decay rate at the squared ME level does not vanish for vanishing $m_{N}$.

\section{B. $W$ boson decays with $|\Delta L|=2$}

Moving to the $L$-violating analogue of the process in Eq. (4.2), we have the 4-body $W$ boson decay chain

$$
\begin{aligned}
W_{\lambda_{W}}^{+}\left(p_{W}\right) & \rightarrow \ell_{R 1}^{+}\left(p_{1}\right) N_{\lambda_{N}}\left(p_{N}\right) \\
& \rightarrow \ell_{R 1}^{+}\left(p_{1}\right) \ell_{R 2}^{+}\left(p_{2}\right) \bar{c}_{R}\left(p_{c}\right) s_{L}\left(p_{s}\right),
\end{aligned}
$$

as shown as a subprocess in Fig. 1(b). Following the same procedure as needed to construct $\mathcal{M}_{L}^{W}$ in Eq. (4.3), the $\mathrm{ME}$ of the $L$-violating decay process is given by

$$
\begin{aligned}
-i \mathcal{M}_{\not}^{W}= & \varepsilon_{\mu}\left(p_{W}, \lambda_{W}\right) T_{\not}^{\nu \mu}\left(p_{1}, p_{2}, p_{1}+p_{c}+p_{s}\right) \\
& \times \Delta_{\nu \rho}\left(p_{c}+p_{s}\right) J^{\rho}\left(p_{s}, p_{c}\right)+\left(p_{1} \leftrightarrow p_{2}\right) .
\end{aligned}
$$

Up to external momentum reassignments, the quark current $J^{\rho}$, polarization vector $\varepsilon_{\mu}$, and propagator $\Delta_{\nu \rho}$ are the same as in the $L$-conserving case. In the last line is the interference from $\ell_{1} \leftrightarrow \ell_{2}$ particle exchange. Due to charge conservation, no second term exists in $\mathcal{M}_{L}^{W}$.

The key difference from the $L$-conserving $\mathrm{ME}$ is the $L$-violating $\left(\ell_{1}^{+} N \ell_{2}^{+}\right)$fermion current. To derive this we note that in going from the $W^{+} \rightarrow \ell_{1}^{+} \ell_{2}^{-} c \bar{s}$ process to $W^{+} \rightarrow \ell_{1}^{+} \ell_{2}^{+} \bar{c} s$, one effectively imposes a charge inversion on the electrically neutral $\left(\ell_{2}^{-} c \bar{s}\right)$ system. Under $C P T$, this is the same as a parity-time inversion and, significantly, is expressible as Feynman rules [79,80].

These state that after assuming a fermion flow [curve in Fig. 1(b)] the $\left(\mathrm{N}_{2}^{+} \mathrm{W}^{-}\right)$vertex as derived from the Lagrangian in Eq. (2.4) is parity-inverted and becomes

$$
-\frac{i g_{W}}{\sqrt{2}} V_{\ell_{2} N} \gamma^{\nu} P_{L} \rightarrow(-1)^{2} \frac{i g_{W}}{\sqrt{2}} V_{\ell_{2} N} \gamma^{\nu} P_{R}
$$

\footnotetext{
${ }^{3}$ We note that the dependence of $\sum\left|\mathcal{M}_{L}^{W}\right|^{2}$ here on $M_{W}^{2}$ does not account for contributions from $\varepsilon_{\mu}, \Delta_{\nu \rho}$, and $J^{\rho}$ in Eq. (4.3). Throughout this entire section we suppress these extra factors.
}

Consistently, as $\ell_{2}$ 's own fermion number flow, which points inward, is antiparallel to the conventional fermion flow, which points outward, its spinor is time-inverted:

$$
\bar{v}_{R}\left(p_{2}\right) \rightarrow \bar{u}_{R}\left(p_{2}\right)
$$

Propagating these modifications and defining for compactness $\tilde{p}_{k} \equiv p_{k}+p_{c}+p_{s}$, for $k=1,2$, we find that both $L$-violating $\left(\ell_{1}^{+} N \ell_{2}^{+}\right)$fermion currents are given by

$$
\begin{gathered}
T_{\psi}^{\nu \mu}\left(p_{1}, p_{2}, \tilde{p}_{k}\right)=-\left(\frac{-i g_{W}}{\sqrt{2}}\right)^{2} V_{\ell_{1} N}^{*} V_{\ell_{2} N} \times \mathcal{N}_{\psi}^{\nu \mu} \times \mathcal{D}\left(\tilde{p}_{k}^{2}\right), \\
\mathcal{N}_{\psi}^{\nu \mu}=\left[\bar{u}_{R}\left(p_{2}\right) \gamma^{\nu} P_{R}\left(\tilde{p}_{k}+m_{N} \mathbb{I}_{4}\right) \gamma^{\mu} P_{L} v_{R}\left(p_{1}\right)\right] .
\end{gathered}
$$

Importantly, this differs from the $L$-conserving analogue $\mathcal{N}_{L}^{\nu \mu}$ in Eq. (4.7) by the replacement of the leftmost chiral projection operator $P_{L}$ with the RH projector $P_{R}$, a consequence of Eq. (4.23). Using again the orthogonality of projection operators we see that the intermediate $N$ is confined to its RH helicity state (the $m_{N} \mathbb{I}_{4}$ term). The $L$-violating transition is helicity inverting as LH helicity states (the $\not \not_{N}$ term) do not contribute to successive chiral interactions of opposite chirality.

After anticommuting the operator $P_{R}$, we obtain

$$
\begin{aligned}
\mathcal{N}_{\not}^{\nu \mu} & =\left[\bar{u}_{R}\left(p_{2}\right) \gamma^{\nu} P_{R}\left(\tilde{p}_{k}+m_{N} \mathbb{I}_{4}\right) \gamma^{\mu} P_{L} v_{R}\left(p_{1}\right)\right] \\
& =m_{N} \times\left[\bar{u}_{L}\left(p_{2}\right) \gamma^{\nu} \gamma^{\mu} P_{L} v_{R}\left(p_{1}\right)\right] \\
& \sim m_{N} \sqrt{E_{2}} \sqrt{E_{1}} \sim m_{N} M_{W} .
\end{aligned}
$$

In the last line we again employ naïve power counting to find that both $\left(\ell_{1}^{+} N \ell_{2}^{+}\right)$currents are proportional to $m_{N}$, independent of $\tilde{p}_{k}$. Subsequently, we see that both currents vanish for vanishing Majorana neutrino mass, in line with expectations from the confusion theorem.

To address the pole structure in the Majorana neutrino's propagator [D in Eq. (4.8)] as we did for the $L$-conserving decay, we consider again when $N$ is (nearly) on-shell. Without the loss of generality, we assume $\tilde{p}_{1}^{2}=\left(p_{1}+p_{c}+p_{s}\right)^{2}$ satisfies the near on-shell condition of Eq. (4.15). By momentum conservation, the nonresonant momentum configuration has the virtuality

$$
\tilde{p}_{2}^{2}=\left(p_{W}-p_{1}\right)^{2}=M_{W}^{2}-2 M_{W} E_{1} .
$$

For these configurations of $\tilde{p}_{k}$, we obtain the expansions

$$
\begin{aligned}
\mathcal{D}\left(\tilde{p}_{1}\right) & =\frac{i}{\left(\tilde{p}_{1}^{2}-m_{N}^{2}\right)+i\left(\Gamma_{N} m_{N}\right)} \\
& =\frac{1}{\Gamma_{N} m_{N}}\left[1-\mathcal{O}\left(\frac{\delta p_{N}^{2}}{\Gamma_{N} m_{N}}\right)\right],
\end{aligned}
$$




$$
\begin{aligned}
\mathcal{D}\left(\tilde{p}_{2}\right) & =\frac{i}{\left(\tilde{p}_{2}^{2}-m_{N}^{2}\right)+i\left(\Gamma_{N} m_{N}\right)} \\
& =\frac{i}{M_{W}^{2}\left(1-2 \frac{E_{1}}{M_{W}}-\frac{m_{N}^{2}-i\left(\Gamma_{N} m_{N}\right)}{M_{W}^{2}}\right)} \\
& =\frac{i}{M_{W}^{2}}\left[1+\mathcal{O}\left(\frac{E_{1}}{M_{W}}\right)+\mathcal{O}\left(\frac{m_{N}^{2}}{M_{W}^{2}}\right)\right] .
\end{aligned}
$$

After combining $\mathcal{D}\left(\tilde{p}_{k}\right)$ with $\mathcal{N}_{\not}^{\nu \mu}$, we see that the $m_{N}$ dependence in the nonresonant contribution scales as

$$
\mathcal{N}_{\not}^{\nu \mu} \times \mathcal{D}\left(\tilde{p}_{2}\right) \sim i \frac{m_{N}}{M_{W}}\left[1+\mathcal{O}\left(\frac{E_{1}}{M_{W}}, \frac{m_{N}^{2}}{M_{W}^{2}}\right)\right],
$$

and thereby vanishes in the limit that $\left(m_{N} / M_{W}\right) \rightarrow 0$. On the other hand, for the resonant contribution, we obtain a qualitatively different behavior, namely that

$$
\mathcal{N}_{\not}^{\nu \mu} \times \mathcal{D}\left(\tilde{p}_{1}\right) \sim \frac{M_{W}}{\Gamma_{N}}\left[1-\mathcal{O}\left(\frac{\delta p_{N}^{2}}{\Gamma_{N} m_{N}}\right)\right] .
$$

This shows that the dependence on N's mass cancels in the resonant contribution and hence generates a nonzero $\mathrm{ME}$ for $W^{+} \rightarrow \ell_{1}^{+} \ell_{2}^{+} \bar{c} s$, even for vanishing $m_{N}$. While helicity inversion exists at the $\mathrm{ME}$ level, its impact is mitigated by the propagator in the on-shell limit, i.e., when $N$ can be approximated as an asymptotic state. Notably, this is independent of active-sterile mixing.

Moreover, since the $\mathrm{ME}$ for $W^{+} \rightarrow \ell_{1}^{+} \ell_{2}^{+} \bar{c} s$ scales as the $\left(\ell_{1}^{+} N \ell_{2}^{+}\right)$current and its crossing interference,

$$
\mathcal{M}_{\not L}^{W} \sim\left[\mathcal{N}_{\not}^{\nu \mu} \times \mathcal{D}\left(\tilde{p}_{1}\right)+\mathcal{N}_{\not}^{\nu \mu} \times \mathcal{D}\left(\tilde{p}_{2}\right)\right]
$$

we find that the resonant, interference, and nonresonant terms respectively contribute to the squared $\mathrm{ME}$ as

$$
\left|\mathcal{M}_{\psi}^{W}\right|^{2} \sim \underbrace{\mathcal{O}\left(\frac{M_{W}^{2}}{\Gamma_{N}^{2}}\right)}_{\text {resonant }}+\underbrace{\mathcal{O}\left(\frac{m_{N}}{\Gamma_{N}}\right)}_{\text {interference }}+\underbrace{\mathcal{O}\left(\frac{m_{N}^{2}}{M_{W}^{2}}\right)}_{\text {nonres. }}
$$

This tells us that while the non-resonant contribution is negligible compared to the (leading) resonant contribution, the (subleading) interference is not guaranteed to be negligible if $m_{N} \sim M_{W}$. However, for $m_{N} \ll M_{W}$, the total width of $N$ scales as $\Gamma_{N} \sim G_{F}^{2} m_{N}^{5}\left|V_{\ell N}\right|^{2}$, and suggests a numerically insignificant interference term.

Using Eq. (4.37) to keep track of formally subleading terms, one finds a more exact scaling of the squared ME:

$$
\begin{aligned}
\sum\left|\mathcal{M}_{\not}^{W}\right|^{2} \sim & \frac{M_{W}^{2}}{\Gamma_{N}^{2}}\left[1-\mathcal{O}\left(\frac{\delta p_{N}^{4}}{\left(\Gamma_{N} m_{N}\right)^{2}}\right)\right] \\
+ & \mathcal{O}\left(\frac{m_{N}}{\Gamma_{N}}\right)+\mathcal{O}\left(\frac{m_{N}^{2}}{M_{W}^{2}}\right) .
\end{aligned}
$$

In comparison to the squared ME in Eq. (4.19), the above demonstrates that in the limit that the Majorana neutrino goes on-shell, the leading contribution to the squared $\mathrm{ME}$ of the $L$-violating decay $W^{+} \rightarrow \ell_{1}^{+} \ell_{2}^{+} \bar{c} s$ admits a dependence on the mass $m_{N}$ that is identical to that found in the $L$-conserving decay $W^{+} \rightarrow \ell_{1}^{+} \ell_{2}^{-} c \bar{s}$.

Furthermore, for both decay processes, the respective contributions from the polarization vector $\varepsilon_{\mu}$, the $W^{\mp}$ propagator $\Delta_{\nu \rho}$, and the $(c \bar{s}) /(\bar{c} s)$ current $J^{\rho}$ in Eqs. (4.3) and (4.22) are the same. It follows that the squared MEs for the two processes do not just have the same scaling dependence on $m_{N}$ and $\Gamma_{N}$ but are, in fact, equal in the limit that $N$ goes on-shell, up to off-shell and finite width corrections. Therefore, after phase space integration, one can anticipate highly comparable decay rates despite the relative presence of helicity inversion.

\section{W boson decays with off-shell $N$}

An important qualification for the above result is Eq. (4.15), which stipulates that the internal Majorana neutrino in the $1 \rightarrow$ 4-body decay is or nearly is on its mass shell. Indeed, when comparing the $L$-conserving and $L$ violating squared MEs in Eqs. (4.19) and (4.40), one sees that the dependence on the neutrino's mass and width only match in this limit. Outside this kinematic limit, mismatches emerge. While a systematic investigation of offshell Majorana neutrinos in $\Delta L=0$ and $|\Delta L|=2$ processes is outside the scope of the present work, we can nevertheless outline some generic features.

If $N$ couples to additional new particles, for example to new Higgs or gauge bosons [7,13,17-20], then its width can be much larger than anticipated by the Lagrangian in Sec. II. In particular, if $N$ is light but has a width comparable to its mass, then the "on-shell" condition,

$$
\delta p_{N}^{2} \equiv\left|p_{N}^{2}-m_{N}^{2}\right| \ll \Gamma_{N} m_{N} \sim m_{N}^{2}<M_{W}^{2},
$$

only weakly constraints the virtuality of the internal Majorana neutrino. By taking the difference $\left(\Delta_{\mathcal{M}}^{\text {Large Width }}\right)$ of the leading contributions in Eqs. (4.19) and (4.40),

$$
\begin{aligned}
& \Delta_{\mathcal{M}}^{\text {Large Width }} \equiv \sum\left|\mathcal{M}_{L}^{W}\right|^{2}-\sum\left|\mathcal{M}_{\psi}^{W}\right|^{2} \\
& \sim \frac{M_{W}^{2}}{\Gamma_{N}^{2}}\left(\frac{p_{N}^{2}}{m_{N}^{2}}-1\right) \times\left[1-\mathcal{O}\left(\frac{\delta p_{N}^{4}}{\left(\Gamma_{N} m_{N}\right)^{2}}\right)\right],
\end{aligned}
$$

a nonzero resultant emerges an grows with the ratio of Majorana neutrino's virtuality $\left(\sqrt{p_{N}^{2}}\right)$ over its mass. For 
virtualities larger than $m_{N}$, there is an enhancement of the $\Delta L=0$ transition probability over the $|\Delta L|=2$ mode, i.e., $\Delta_{\mathcal{M}}^{\text {Large Width }}>0$. We trace this to the $L$-conserving $\left(\ell_{1}^{+} N \ell_{2}^{-}\right)$ lepton current, which as shown in Eq. (4.11), selects for the LH helicity of $N$ and is thus proportional to its momentum $\left(\not \not_{N}\right)$. In the $L$-violating case, the $\left(\ell_{1}^{+} N \ell_{2}^{+}\right)$current selects for the RH helicity of $N$ and thus is proportional to its mass $\left(m_{N} \mathbb{I}_{4}\right)$. For virtualities smaller than $m_{N}$, the relative helicity enhancement/suppression is inverted with $\Delta_{\mathcal{M}}^{\text {Large Width }}<0$.

In an altogether different limit, it may be that $N$ is too heavy to ever be resonantly produced in $W$ boson decays. In this case, one enters the decoupling limit [84] and the pole structure of $N$ 's propagator behaves as

$$
\begin{aligned}
\mathcal{D}\left(\tilde{p}_{k}\right) & =\frac{i}{\left(\tilde{p}_{k}^{2}-m_{N}^{2}\right)+i\left(\Gamma_{N} m_{N}\right)} \\
& =\frac{-i}{m_{N}^{2}}\left[1+\mathcal{O}\left(\frac{\tilde{p}_{k}^{2}}{m_{N}^{2}}\right)+i \mathcal{O}\left(\frac{\Gamma_{N}}{m_{N}}\right)\right] .
\end{aligned}
$$

Importantly, the propagator's leading contribution is the same for the $\Delta L=0 \mathrm{ME}$ as well as both diagrams in the $|\Delta L|=2$ ME. Thus, any difference between the two transition rates is ultimately due to helicity inversion.

After propagating this expansion, the squared MEs for the $L$-conserving and $L$-violating $W$ boson decays are

$$
\begin{gathered}
\sum\left|\mathcal{M}_{L}^{W}\right|^{2} \sim \frac{M_{W}^{2} p_{N}^{2}}{m_{N}^{4}}\left[1+\mathcal{O}\left(\frac{p_{N}^{2}}{m_{N}^{2}}, \frac{\Gamma_{N}}{m_{N}}\right)\right], \\
\sum\left|\mathcal{M}_{\not}^{W}\right|^{2} \sim \frac{M_{W}^{2}}{m_{N}^{2}}\left[1+\mathcal{O}\left(\frac{p_{N}^{2}}{m_{N}^{2}}, \frac{\Gamma_{N}}{m_{N}}\right)\right] .
\end{gathered}
$$

Likewise, their difference $\left(\Delta_{\mathcal{M}}^{\text {Large Mass }}\right)$ is given by

$$
\begin{aligned}
\Delta_{\mathcal{M}}^{\text {Large Mass }} & \equiv \sum\left|\mathcal{M}_{L}^{W}\right|^{2}-\sum\left|\mathcal{M}_{\not}^{W}\right|^{2} \\
& \sim \frac{M_{W}^{2}}{m_{N}^{2}}\left(\frac{p_{N}^{2}}{m_{N}^{2}}-1\right) \times\left[1+\mathcal{O}\left(\frac{p_{N}^{2}}{m_{N}^{2}}, \frac{\Gamma_{N}}{m_{N}}\right)\right] .
\end{aligned}
$$

Immediately, we see that the $L$-conserving case exhibits a quartic dependence on the Majorana neutrino's mass, whereas the $L$-violating case has only a quadratic dependence. This reveals that in the decoupling limit the transition rate for the $\Delta L=0$ process vanishes faster than the $|\Delta L|=2$ transition rate. In the language of effective field theories, this is the manifestation of $L$-conserving operators at dimension eight decoupling more quickly than $L$-violating operators at dimension seven.

As in the large-width scenario, the difference between the two squared MEs stems from the respective preservation and inversion of helicity in the $\left(\ell_{1}^{+} N \ell_{2}^{-}\right)$and $\left(\ell_{1}^{+} N \ell_{2}^{+}\right)$ lepton currents. More specifically, the $m_{N}$ factor that is collected in the $|\Delta L|=2$ case partially compensates the mass suppression in Eq. (4.45), and reduces the dimension of $L$-violating operators. This is unlike the large-width scenario, where the virtuality of $N$ can exceed its mass and leads to an enhancement of the $L$-conserving transition. The virtuality of $N$ in the decoupling limit is always smaller than its mass and therefore leads to a suppression of the $L$-conserving transition.

\section{D. $2 \rightarrow 4$ scattering with $\Delta L=0$ and $|\Delta L|=2$}

To extrapolate our findings, i.e., the existence of helicity inversion but the absence of helicity suppression in $L$ violating decays of $W$ bosons involving (nearly) on-shell Majorana $N$, to other processes, it is helpful to stress that the above arguments are kinematical in nature. They rely on Lorentz invariance, spin correlation, and expansions around leading regions of phase space. They do not rely on strong interference, flavor symmetries, or mixing suppression that one often encounters [31-34,60-62]. As such, the results are process-dependent and are sensitive to whether Eq. (4.15), or a similar relation, is satisfied.

With this in mind, one direction where it is possible to extrapolate the above phenomenon is to $2 \rightarrow n$ scattering processes. In particular, there is the $L$-conserving,

$$
\begin{aligned}
u_{L}\left(p_{u}\right) \bar{d}_{R}\left(p_{d}\right) & \rightarrow W_{\lambda_{W}}^{+}\left(p_{W}\right) \rightarrow \ell_{1 R}^{+}\left(p_{1}\right) N_{\lambda_{N}}\left(p_{N}\right) \\
& \rightarrow \ell_{1 R}^{+}\left(p_{1}\right) \ell_{L 2}^{-}\left(p_{2}\right) c_{L}\left(p_{c}\right) \bar{s}_{R}\left(p_{s}\right),
\end{aligned}
$$

as shown in Fig. 1(a), and the $L$-violating,

$$
\begin{aligned}
u_{L}\left(p_{u}\right) \bar{d}_{R}\left(p_{d}\right) & \rightarrow W_{\lambda_{W}}^{+}\left(p_{W}\right) \rightarrow \ell_{1 R}^{+}\left(p_{1}\right) N_{\lambda_{N}}\left(p_{N}\right) \\
& \rightarrow \ell_{1 R}^{+}\left(p_{1}\right) \ell_{R 2}^{+}\left(p_{2}\right) \bar{c}_{R}\left(p_{c}\right) s_{L}\left(p_{s}\right),
\end{aligned}
$$

as shown in Fig. 1(b). The novelty of these channels follows from the limitations in the $W$ decay case study. The first limitation relates to the idealization of working with an unpolarized, on-shell $W$ boson. This is an object that is never really actualized in nature. By virtue of the $W$ 's chiral couplings, real $W \mathrm{~s}$ are produced with some degree of polarization [85-87]. Likewise, a degree of off-shell virtuality is nearly always present and such contributions are not guaranteed to be negligible if $m_{N} \sim M_{W}$ [46-49].

To check the impact of these matters on the existence of inversion and suppression in Eqs. (4.50)-(4.51), we again construct the associated MEs. These can be built from the MEs in Eqs. (4.3) and (4.22) for a $W$ decay to an approximately on-shell $N$ by working in the hard scattering frame with the following momentum assignments

$$
\begin{aligned}
& p_{u}=\frac{Q}{2}(1,0,0,1), \quad p_{d}=\frac{Q}{2}(1,0,0,-1), \\
& p_{W}=p_{1}+p_{2}, \quad Q^{2}=p_{W}^{2}=\left(p_{1}+p_{2}\right)^{2} .
\end{aligned}
$$


After substituting the $W$ polarization vector for the current and propagator of the sub-process $u_{L} \bar{d}_{R} \rightarrow W^{+*}$,

$$
\varepsilon_{\mu}\left(p_{W}\right) \rightarrow \tilde{J}^{\sigma}\left(p_{u}, p_{d}\right) \Delta_{\sigma \mu}\left(p_{W}=p_{u}+p_{d}\right),
$$

where the propagator $\Delta$ is the same as in Eq. (4.5) and the $\left(u_{L} \bar{d}_{R}\right)$ current $\tilde{J}$ is given by

$$
\tilde{J}^{\sigma}\left(p_{u}, p_{d}\right)=\frac{-i g_{W} \delta^{A B}}{\sqrt{2}}\left[\bar{v}_{R}^{B}\left(p_{d}\right) \gamma^{\sigma} P_{L} u_{L}^{A}\left(p_{u}\right)\right],
$$

one obtains the following MEs for the $L$-conserving $\left(\mathcal{M}_{L}^{(2 \rightarrow 4)}\right)$ and $L$-violating $\left(\mathcal{M}_{\psi}^{(2 \rightarrow 4)}\right)$ scattering processes:

$$
\begin{aligned}
-i \mathcal{M}_{L}^{(2 \rightarrow 4)}= & \tilde{J}^{\sigma}\left(p_{u}, p_{d}\right) \Delta_{\sigma \mu}\left(p_{u}+p_{d}\right) T_{L}^{\nu \mu}\left(p_{1}, p_{2}, p_{N}\right) \\
& \times \Delta_{\nu \rho}\left(p_{c}+p_{s}\right) J^{\rho}\left(p_{c}, p_{s}\right), \\
-i \mathcal{M}_{\psi}^{(2 \rightarrow 4)}= & \tilde{J}^{\sigma}\left(p_{u}, p_{d}\right) \Delta_{\sigma \mu}\left(p_{u}+p_{d}\right) \\
& \times T_{\not \psi}^{\nu \mu}\left(p_{1}, p_{2}, p_{1}+p_{c}+p_{s}\right) \\
& \times \Delta_{\nu \rho}\left(p_{c}+p_{s}\right) J^{\rho}\left(p_{s}, p_{c}\right)+\left(p_{1} \leftrightarrow p_{2}\right) .
\end{aligned}
$$

To extract the scaling behavior of these two MEs, we exploit the fact that the $W$ 's longitudinal polarization $\left(\lambda_{W}=0\right)$, which generates a different mass-energy power counting than transverse polarizations $\left(\lambda_{W}= \pm 1\right)$, does not couple to massless fermions. It does not contribute to the $u \bar{d} \rightarrow W^{*} \rightarrow N \ell$ subprocess, regardless of external polarizations. Using this and after explicit evaluation of the helicity spinor algebra, we obtain for both cases,

$$
\begin{aligned}
& \tilde{J}^{\sigma}\left(p_{u}, p_{d}\right) \Delta_{\sigma \mu}\left(p_{u}+p_{d}\right) \\
& =(-i)^{2} \delta^{A B} \frac{g_{W}}{\sqrt{2}} \frac{\left[\bar{v}_{R}^{B}\left(p_{d}\right) \gamma_{\mu} P_{L} u_{L}^{A}\left(p_{u}\right)\right]}{\left(Q^{2}-M_{W}^{2}+i \Gamma_{W} M_{W}\right)} \\
& =(-i)^{2} \delta^{A B} \frac{g_{W}}{\sqrt{2}} \frac{Q(0,1,-i, 0)}{\left(Q^{2}-M_{W}^{2}+i \Gamma_{W} M_{W}\right)} \\
& \quad \sim \frac{Q}{\Gamma_{W} M_{W}}\left[1-\mathcal{O}\left(\frac{\left(Q^{2}-M_{W}^{2}\right)}{\Gamma_{W} M_{W}}\right)\right] .
\end{aligned}
$$

For clarity, we expanded the $W$ 's propagator in the final line around its on-shell limit, i.e., $\left(Q^{2}-M_{W}^{2}\right) \ll \Gamma_{W} M_{W}$.

It is evident that the substitution in Eq. (4.54) does not introduce additional parity inversion, say via coupling to longitudinal modes, nor any new dependence on $m_{N}$. As a consequence, the scaling behavior of the $\left(\ell_{1}^{+} N \ell_{2}^{\mp}\right)$ lepton currents and propagators in the scattering process are the same as in the decay process, up to substitutions of the total c.m. energy: $M_{W} \rightarrow Q$. Consistently, this means external momenta scale as $E_{\text {external }} \sim Q$.

Propagating these modifications, one finds that in the double on-shell limit, the leading contributions to the squared MEs for the $2 \rightarrow 4$ processes scale as

$$
\begin{aligned}
& \sum\left|\mathcal{M}_{L}^{(2 \rightarrow 4)}\right|^{2} \\
& \sim \frac{Q^{4} p_{N}^{2}}{\left(\Gamma_{W} M_{W}\right)^{2}\left(\Gamma_{N} m_{N}\right)^{2}} \\
& \quad \times\left[1-\mathcal{O}\left(\frac{\left(Q^{2}-M_{W}^{2}\right)}{\Gamma_{W} M_{W}}\right)-\mathcal{O}\left(\frac{\delta p_{N}^{4}}{\left(\Gamma_{N} m_{N}\right)^{2}}\right)\right], \\
& \sum\left|\mathcal{M}_{\not}^{(2 \rightarrow 4)}\right|^{2} \\
& \sim \frac{Q^{4}}{\left(\Gamma_{W} M_{W}\right)^{2} \Gamma_{N}^{2}} \\
& \quad \times\left[1-\mathcal{O}\left(\frac{\left(Q^{2}-M_{W}^{2}\right)}{\Gamma_{W} M_{W}}\right)-\mathcal{O}\left(\frac{\delta p_{N}^{4}}{\left(\Gamma_{N} m_{N}\right)^{2}}\right)\right] .
\end{aligned}
$$

As in the $1 \rightarrow 4$ decays, we find that the helicity inversion in $2 \rightarrow 4$ scattering does not manifest as helicity suppression in the on-shell limit for $N$ when $\Gamma_{N} \ll m_{N}$. In fact, we find again that the squared $\mathrm{ME}$ for the $L$-conserving and $L$-violating processes are the same, up to the heavy neutrino's off-shellness. Thus, one obtains equal cross sections in the absence of phase space cuts. This lack of helicity suppression/enhancement for off-shell gauge mediators is consistent with past studies on related phenomena $[35,38,88]$. When $N$ is dominantly off-shell, the same enhancements/suppressions described in Sec. IV C appear, up to appropriate $M_{W} \rightarrow Q$ substitutions.

Following analogous arguments, we anticipate that these findings hold also for cascade decay processes, such as top quark decays to Majorana neutrinos, $t \rightarrow b \ell_{1} N \rightarrow$ $b \ell_{1} \ell_{2} f_{1} \overline{f_{2}}$ [89-91]. In such situations, contributions from the $W$ 's longitudinal polarization may introduce additional dependencies on $\left(Q / M_{W}\right) \sim\left(m_{t} / M_{W}\right)$ but otherwise not alter the tensor structure of the $\left(\ell_{1} N \ell_{2}\right)$ lepton currents. As such, the scaling of squared momenta for $N$ will remain the same in its near on-shell limit.

As a brief remark, we note that at next-to-leading order in EW it may be that differences in the $L$-conserving and $L$ violating processes generate asymmetric transition rates. Likewise, while virtual $\mathcal{O}\left(\alpha_{s}\right)$ corrections to the processes in Fig. 1 will not impact the polarization of the intermediate $N$ [92], the expectation for real $\mathcal{O}\left(\alpha_{s}\right)$ emissions is less clear. In principle, these effects are coupling-suppressed but such considerations are left for the future.

\section{E. Other scenarios with $|\Delta L|=\mathbf{2}$}

A second direction where one can apply the above findings is to other new physics scenarios that feature chiral gauge interactions and Majorana fermions. While a 
systematic survey is beyond the present scope of this work, two concrete examples are: warped extra dimension with RH Majorana neutrinos $\left(\nu_{R}\right)$ [93-95] and the left-right symmetric model (LRSM) [7,13,17-20].

The first is characterized by Kaluza-Klein (KK) excitations of SM particles as well as of $\nu_{R}$. This includes, for example, $W_{K K}^{\prime}$ gauge bosons, which have the same chiral interaction structure and gauge quantum numbers as the SM $W$ boson. After mass-diagonalization, the resulting Lagrangian that governs interactions between the mass eigenstates $N_{K K}, W_{K K}^{\prime \pm}$, and $\ell_{K K}^{ \pm}$is essentially the same as Eq. (2.4), up to an overall rescaling of couplings.

Phenomenologically speaking, this allows processes like those shown in Fig. 1 but with internal particles substituted with their KK excitations. Corresponding MEs and squared MEs are therefore the same as those constructed in Secs. IV A-IV D, up to substitutions of mass and coupling constants, implying the presence of helicity inversion. So long as external particles are massless and the near on-shell condition of Eq. (4.15) is satisfied, one should consistently find an absence of helicity suppression, modulo off-shell virtuality and finite width effects.

In the second case, the LRSM is characterized by embedding the SM's $\mathcal{G}_{\mathrm{SM}}=S U(3)_{c} \otimes S U(2)_{L} \otimes U(1)_{Y}$ gauge symmetry into the larger symmetry group, $\mathcal{G}_{\text {LRSM }}=$ $S U(3)_{c} \otimes S U(2)_{L} \otimes S U(2)_{R} \otimes U(1)_{B-L} \otimes \mathcal{P} . \quad$ In this model, all of the SM's RH chiral fields and $\nu_{R}$ are charged under the $S U(2)_{R}$ gauge group, just as their LH counter parts are charged under $S U(2)_{L}$. The $U(1)$ conservation of baryon-minus-lepton numbers $(B-L)$ ensures that the theory is anomaly free and the generalized discrete parity $\mathcal{P}$ ensures that the $\mathrm{LH}$ and $\mathrm{RH}$ gauge interactions are identical before spontaneous symmetry breaking.

After LR and EW symmetry breaking, one finds RH gauge bosons $W_{R}$ that couple to heavy Majorana neutrinos $N$ and charged leptons $\ell$ through RH chiral currents, in analogy to the Lagrangian in Eq. (2.4). This leads to the spectacular $L$-violating scattering process [44]

$$
\begin{aligned}
u_{R} \bar{d}_{L} & \rightarrow W_{R}^{+} \rightarrow \ell_{1 L}^{+} N \rightarrow \ell_{1 L}^{+} \ell_{L 2}^{+} W_{R}^{-*} \\
& \rightarrow \ell_{1 L}^{+} \ell_{L 2}^{+} \bar{c}_{L} s_{R} .
\end{aligned}
$$

This associated diagram is the same as Fig. 1(b) but with substituting the SM gauge boson $W$ for LRSM gauge boson $W_{R}$. Explicit computation reveals a ME that is identical to the $L$-violating ME of Eq. (4.57), up to substitutions of masses and couplings as well as the exchange of $P_{L}$ chiral projection operators for the operator $P_{R}$.

Importantly, the consistent application of the Feynman rules of Refs. $[79,80]$ requires the vertex modifications

$$
\begin{aligned}
& \gamma^{\nu} P_{R} \rightarrow(-1) \gamma^{\nu} P_{L} \\
& \bar{v}_{L}\left(p_{2}\right) \rightarrow \bar{u}_{L}\left(p_{2}\right) .
\end{aligned}
$$

This leads to an explicit helicity inversion at the ME level as in the phenomenological type I seesaw. Assuming that the near on-shell condition for intermediate resonances is satisfied, one again finds that the explicit dependence on $m_{N}^{2}$ cancels. Again, this leads to an absence of helicity suppression, up to the now-usual caveats.

For completeness, one could also consider the mixed $W_{R}-W_{L}$ scattering process given by [35]

$$
\begin{aligned}
u_{R} \bar{d}_{L} & \rightarrow W_{R}^{+} \rightarrow \ell_{1 L}^{+} N \rightarrow \ell_{1 L}^{+} \ell_{R 2}^{+} W_{L}^{-*} \\
& \rightarrow \ell_{1 L}^{+} \ell_{R 2}^{+} \bar{c}_{R} s_{L} .
\end{aligned}
$$

In this case, one finds a second helicity inversion due to inverting the chiral coupling associated with the second charged current. This implies that the roles are now reversed: the $L$-violating process exhibits a net helicity conservation while the $L$-conserving process exhibits a net helicity inversion. Explicit calculation [35] again shows a lack of helicity suppression in the near on-shell limit.

\section{NUMERICAL IMPACT OF HELICITY INVERSION IN $|\Delta L|=2$ LHC PROCESSES}

In light of the previous section, the question is not whether there is helicity inversion in $|\Delta L|=2$ amplitudes mediated by Majorana neutrinos in the phenomenological type I seesaw. It exists and follows from a parity inversion in EW interactions. The pertinent issue is whether contributions from off-shell virtualities, which can give rise to helicity-suppressing behavior, is numerically relevant for searches for Majorana $N$ at the LHC.

To investigate this, we consider two complementary measures of helicity suppression. The first, presented in Sec. VC, is based the potential asymmetry that could develop in $L$-conserving and $L$-violating decays of the SM $W$ boson. The second, presented in Sec. VD, is the analogous asymmetry that can appear in hadronic $2 \rightarrow 4$ cross sections. For both cases we inherently work in a limit where resonant production of $N$ dominates. Therefore in Sec. V E we investigate the possible importance of off-shell contributions. Before presenting our numerical results, we comment in Sec. VA on the preservation of spin-correlation in our computations and then validate the presence of strong helicity inversion in Sec. V B.

\section{A. Numerical preservation of spin-correlation}

To undertake our numerical computations we exploit the massive spinor helicity formalism of Refs. [71-74] as implemented in the ALOHA package $[53,68]$, in the HELAS basis [72]. (For precise details of the computational setup, see Sec. III.) We do so in order to evaluate MEs exactly but at the cost of analytical expressions.

We forego analytical expressions due to the fact that we are dealing with multiscale, $1 \rightarrow 4$ and $2 \rightarrow 4$ processes. The squared MEs for these processes must be amended 
TABLE I. Helicity amplitudes for the $W^{+}\left(\lambda_{W}\right) \rightarrow e^{+}\left(\lambda_{e}\right) N\left(\lambda_{N}\right)$ decay, with kinematics defined in Sec. V B.

\begin{tabular}{lccc}
\hline \hline & \multicolumn{3}{c}{$W^{+}\left(\lambda_{W}\right) \rightarrow e^{+}\left(\lambda_{e}\right) N\left(\lambda_{N}\right)$} \\
\hline$\lambda_{W}$ & $\lambda_{e}$ & $\lambda_{N}$ & $-i \mathcal{M}\left(\lambda_{W}, \lambda_{e}, \lambda_{N}\right) /\left(\frac{-i g_{W}}{\sqrt{2}} V_{e N}\right)$ \\
+1 & $R$ & $R$ & $\frac{1}{\sqrt{2}} m_{N} \sqrt{1-r_{N}} \sin \theta_{e}$ \\
0 & $R$ & $R$ & $-m_{N} \sqrt{1-r_{N}} \cos \theta_{e} e^{-i \phi_{e}}$ \\
-1 & $R$ & $R$ & $-\frac{1}{\sqrt{2}} m_{N} \sqrt{1-r_{N}} \sin \theta_{e} e^{-i 2 \phi_{e}}$ \\
+1 & $R$ & $L$ & $\frac{1}{\sqrt{2}} M_{W} \sqrt{1-r_{N}}\left(1+\cos \theta_{e}\right) e^{i \phi_{e}}$ \\
0 & $R$ & $L$ & $M_{W} \sqrt{1-r_{N}} \sin \theta_{e}$ \\
-1 & $R$ & $L$ & $\frac{1}{\sqrt{2}} M_{W} \sqrt{1-r_{N}}\left(1-\cos \theta_{e}\right) e^{-i \phi_{e}}$ \\
All & $L$ & All & 0 \\
\hline \hline
\end{tabular}

with kinematic factors and integrated over phase space to derive total decay widths $(\Gamma)$ and cross sections $(\sigma)$, i.e., the quantities considered here. In the absence of strong assumptions like the narrow width approximation (NWA), phase space integration usually leaves intractable algebraic expressions for such processes. However, we avoid employing the NWA since its rigorous justification for EW-scale Majorana neutrinos is not well-established in the literature. On the contrary, studies into the validity of the NWA itself list criteria that may not be satisfied here [46-50], and even show [46] a sizable impact on the spin-correlation propagated by Majorana fermions. While important, such considerations are outside our scope and deferred to later work.

\section{B. Numerical validation of helicity inversion}

As a first step to quantifying potential helicity suppression in $|\Delta L|=2$ transitions, we move to establish that our computational setup captures the helicity inversion in such processes. To demonstrate this and in the notation of Sec. IV we consider the simpler $1 \rightarrow 2$ decay

$$
W_{\lambda_{W}}^{+}\left(p_{W}\right) \rightarrow e_{\lambda_{e}}^{+}\left(p_{e}\right) N_{\lambda_{N}}\left(p_{N}\right) .
$$

In the $W$ boson's rest frame and with the assignments,

$$
\begin{gathered}
p_{e}=E_{e}\left(1, \sin \theta_{e} \cos \phi_{e}, \sin \theta_{e} \sin \phi_{e}, \cos \theta_{e}\right), \\
p_{W}=M_{W}(1,0,0,0), \quad E_{e}=\frac{M_{W}}{2}\left(1-r_{N}\right), \\
p_{N}=p_{W}-p_{e}, \quad r_{N} \equiv\left(\frac{m_{N}}{M_{W}}\right)^{2},
\end{gathered}
$$

we evaluate and report the amplitude $\mathcal{M}\left(\lambda_{W}, \lambda_{e}, \lambda_{N}\right)$ for each helicity permutation $\left(\lambda_{W}, \lambda_{e}, \lambda_{N}\right)$ in Table I.

Several notable features can be identified in the MEs of Table I. First is that all amplitudes for $e^{+}\left(\lambda_{e}=L\right)$ are zero, which is consistent with $W$ bosons only coupling to massless LH particles (RH antiparticles). Second is that
TABLE II. Same as Table I but for the $W^{+}\left(\lambda_{W}\right) \rightarrow$ $e^{+}\left(\lambda_{e}\right) N\left(\lambda_{N}\right)$ partial width. Note: the spin-averaging factor of $\mathcal{S}_{W}=3$ is not included in $\Gamma$.

\begin{tabular}{lccc}
\hline \hline & \multicolumn{3}{c}{$W^{+}\left(\lambda_{W}\right) \rightarrow e^{+}\left(\lambda_{e}\right) N\left(\lambda_{N}\right)$} \\
\hline$\lambda_{W}$ & $\lambda_{e}$ & $\lambda_{N}$ & $\Gamma\left(\lambda_{W}, \lambda_{e}, \lambda_{N}\right)$ \\
+1 & $R$ & $R$ & $\frac{g_{W}^{2}}{96 \pi}\left|V_{e N}\right|^{2} m_{N}\left(\frac{m_{N}}{M_{W}}\right)\left(1-r_{N}\right)^{2}$ \\
0 & $R$ & $R$ & $=\Gamma(+, R, R)$ \\
-1 & $R$ & $R$ & $=\Gamma(+, R, R)$ \\
+1 & $R$ & $L$ & $\frac{g_{W}^{2}}{48 \pi}\left|V_{e N}\right|^{2} M_{W}\left(1-r_{N}\right)^{2}$ \\
0 & $R$ & $L$ & $=\Gamma(+, R, L)$ \\
-1 & $R$ & $L$ & $=\Gamma(+, R, L)$ \\
All & $L$ & All & 0 \\
\hline \hline
\end{tabular}

amplitudes for $\lambda_{W}= \pm 1$ and $\lambda_{N}=L$ feature the characteristic $(1 \pm \cos \theta)$ behavior associated with vector currents. Third, and most relevant, is that amplitudes for $\lambda_{N}=R$ scale with the mass of $N$, i.e., $-i \mathcal{M} \sim m_{N}$, whereas amplitudes for $\lambda_{N}=L$ scale with the energy of $N$, i.e., $-i \mathcal{M} \sim E_{N} \sim M_{W}$, as one would expect for helicity inversion of massive decay products.

Using the definition of the partial decay width for the unpolarized particle $\mathcal{B}$ with mass $m_{\mathcal{B}}$ into final-state $f$,

$\Gamma\left(\mathcal{B} \rightarrow f_{\lambda_{f}}\right)=\frac{1}{2 m_{\mathcal{B}} \mathcal{S}_{\mathcal{B}} N_{c}^{\mathcal{B}}} \int d P S_{f} \sum_{\text {dof }}\left|\mathcal{M}\left(\mathcal{B}_{\lambda_{\mathcal{B}}} \rightarrow f_{\lambda_{f}}\right)\right|^{2}$,

we report in Table II the partial width $\Gamma\left(\lambda_{W}, \lambda_{e}, \lambda_{N}\right)$ for each permutation of helicities $\left(\lambda_{W}, \lambda_{e}, \lambda_{N}\right)$. We note that, for consistency, the spin-averaging factor of $\mathcal{S}_{W}=3$ is not included in $\Gamma\left(\lambda_{W}, \lambda_{e}, \lambda_{N}\right)$. This implies that the canonical spin-averaged total is related by

$$
\Gamma\left(W^{+} \rightarrow e^{+} N\right)=\frac{1}{\mathcal{S}_{W}} \sum_{\lambda_{k}} \Gamma\left(W_{\lambda_{W}}^{+} \rightarrow e_{\lambda_{e}}^{+} N_{\lambda_{N}}\right) .
$$

Likewise, the partial and total widths of $W$ are related to its branching rate (BR) by the usual definition

$$
\mathrm{BR}(W \rightarrow f) \equiv \frac{\Gamma(W \rightarrow f)}{\Gamma_{W}}=\frac{\Gamma(W \rightarrow f)}{\sum_{X} \Gamma(W \rightarrow X)} .
$$

In comparison to the MEs, we observe in the partial widths listed in Table II that several kinematic features are washed out after phase space integration. In particular, the characteristic $(1 \pm \cos \theta)$ behavior and sensitivity to the azimuthal angle $\phi_{e}$ are no longer manifest. What remains, however, is the relative dependence on the heavy neutrino's mass. For the $\lambda_{N}=L$ cases, we see that the ME's linear power dependence on $M_{W}$ remains linear in the partial widths. The quadratic power one obtains at the squared ME 


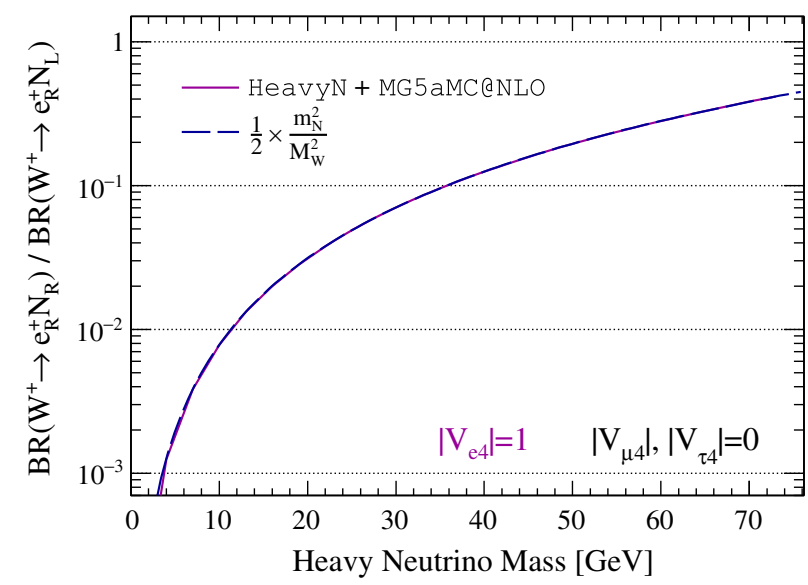

FIG. 2. The $W^{+} \rightarrow e_{R}^{+} N_{R}$ and $W^{+} \rightarrow e_{R}^{+} N_{L}$ branching ratio as a function of $m_{N}$ [GeV], as computed numerically from polarized matrix elements (solid) and analytically (dashed).

level is canceled by the explicit mass factor in the definition of $\Gamma$ in Eq. (5.5). For the $\lambda_{N}=R$ cases, the linear power dependence on $m_{N}$ at the ME level grows at the squared ME level, and leads the partial widths to scale as $\Gamma \sim$ $m_{N}\left(m_{N} / M_{W}\right)$. Interestingly, this shows that in the fixed $m_{N}$ but large $M_{W}$ limit, the MEs for $\lambda_{N}=R$ marginally grow and converge, whereas the partial widths vanish. This behavior is consistent with expectations from the confusion theorem.

In taking the ratio of the $W^{+} \rightarrow e^{+} N_{\lambda_{N}}$ branching rates, we can extract the helicity suppression of $\lambda_{N}=R$ helicity states at small $\left(m_{N} / M_{W}\right)^{2}$, and verify the modeling in our setup. Analytically the ratio is given by

$$
\begin{aligned}
\mathcal{R} & \equiv \frac{\mathrm{BR}\left(W^{+} \rightarrow e_{R}^{+} N_{R}\right)}{\operatorname{BR}\left(W^{+} \rightarrow e_{R}^{+} N_{L}\right)}=\frac{\Gamma\left(W^{+} \rightarrow e_{R}^{+} N_{R}\right)}{\Gamma\left(W^{+} \rightarrow e_{R}^{+} N_{L}\right)} \\
& =\frac{\frac{1}{\mathcal{S}_{W}} \sum_{\lambda} \Gamma\left(W_{\lambda}^{+} \rightarrow e_{R}^{+} N_{R}\right)}{\frac{1}{\mathcal{S}_{W}} \sum_{\lambda} \Gamma\left(W_{\lambda}^{+} \rightarrow e_{R}^{+} N_{L}\right)} \\
& =\frac{1}{2}\left(\frac{m_{N}}{M_{W}}\right)^{2} .
\end{aligned}
$$

In Fig. 2 we plot $\mathcal{R}$ as a function of heavy neutrino mass $m_{N}[\mathrm{GeV}]$ as computed numerically from polarized matrix elements (solid line) and analytically (dashed line). For heavy neutrino masses in the range of $m_{N} \in$ $[1 \mathrm{GeV}, 75 \mathrm{GeV}]$ we find that $\mathcal{R}$ spans $3-4$ orders of magnitude. Over this entire range we find excellent agreement between our numerical setup and exact analytic expectations. This provides nontrivial checks that (i) helicity inversion for viable values of heavy neutrino masses can be numerically significant, and (ii) our computational setup successfully captures such behavior.

Briefly, we note that we do not consider Majorana neutrinos with masses below $m_{N}=1 \mathrm{GeV}$. For such states the relevant virtuality scales are comparable to the nonperturbative scale of QCD. Hence, one should treat the decays of lighter sterile neutrinos, i.e., for $m_{N_{k}} \lesssim 1-10 \mathrm{GeV}$, like decays of $\tau$ leptons and adopt a low-energy, effective field theory, as done for example in Refs. $[25,41,96]$. This introduces additional parity nuances that have been considered elsewhere [41].

\section{Total width asymmetry}

As our first measure of helicity suppression in LHC observables for processes that are mediated by heavy Majorana neutrinos, we consider respectively the $L$-conserving and $L$-violating, $1 \rightarrow 4$-body $W$ boson decay processes,

$$
\begin{gathered}
\Delta L=0: W^{+} \rightarrow e_{1}^{+} N \rightarrow e_{1}^{+} e_{2}^{-} c \bar{s}, \\
|\Delta L|=2: W^{+} \rightarrow e_{1}^{+} N \rightarrow e_{1}^{+} e_{2}^{+} \bar{c} s .
\end{gathered}
$$

Here we fix final-state flavors for definiteness. Diagrams with $\gamma^{*} / Z^{*}$ exchange are removed in a gauge-invariant manner, resulting in those shown in Fig. 1. Interfering diagrams from identical particle exchange are kept.

In Sec. IV, we argued that the ME for these processes exhibit different parametric dependencies on $m_{N}$ due helicity inversion. At the same time we showed in Sec. V B that Lorentz invariance lead to the same parametric dependence in squared MEs, in the on-shell limit for $N$. Differences in decay rates were found to be proportional to the off-shell virtuality of $N$ as well as to its total width. To address the importance of these terms and quantify the existence of any such helicity suppression, we consider the following asymmetry $\mathcal{A}_{\Gamma}$ in branching rates:

$$
\begin{aligned}
\mathcal{A}_{\Gamma} & \equiv \frac{\mathrm{BR}\left(W^{+} \rightarrow e^{+} e^{-} c \bar{s}\right)-\mathrm{BR}\left(W^{+} \rightarrow e^{+} e^{+} \bar{c} s\right)}{\operatorname{BR}\left(W^{+} \rightarrow e^{+} e^{-} c \bar{s}\right)+\mathrm{BR}\left(W^{+} \rightarrow e^{+} e^{+} \bar{c} s\right)} \\
& =\frac{\Gamma\left(W^{+} \rightarrow e^{+} e^{-} c \bar{s}\right)-\Gamma\left(W^{+} \rightarrow e^{+} e^{+} \bar{c} s\right)}{\Gamma\left(W^{+} \rightarrow e^{+} e^{-} c \bar{s}\right)+\Gamma\left(W^{+} \rightarrow e^{+} e^{+} \bar{c} s\right)} \\
& \equiv \frac{\Gamma_{\mathrm{LNC}}-\Gamma_{\mathrm{LNV}}}{\Gamma_{\mathrm{LNC}}+\Gamma_{\mathrm{LNV}}} .
\end{aligned}
$$

In Fig. 3(a) we show the decay rate asymmetry $\mathcal{A}_{\Gamma}$ between the $L$-conserving and $L$-violating $W^{+}$boson decays given in Eqs. (5.11)-(5.12), as a function of $m_{N}$ $[\mathrm{GeV}]$ for representative active-sterile neutrino mixing $\left|V_{e N}\right|^{2}=1$ (solid), $10^{-2}$ (dash-dot), and $10^{-4}$ (dash). Also shown is the associated statistical MC uncertainty band $\left(\delta \mathcal{A}_{\mathrm{MC}}\right)$. Based on $N=100 k$ events per determination of $\Gamma$ we obtain a statistical MC uncertainty that is nearly uniform and is approximately $\delta \mathcal{A}_{\mathrm{MC}} \approx 2.2 \times 10^{-3}$.

For heavy neutrino masses in the range of $m_{N} \in$ [1 GeV, $75 \mathrm{GeV}]$ we report asymmetries consistent with $\mathcal{A}_{\Gamma}=0$, i.e., no asymmetry and hence no helicity suppression. More precisely, we find nonzero $\mathcal{A}_{\Gamma}$ that fluctuate 


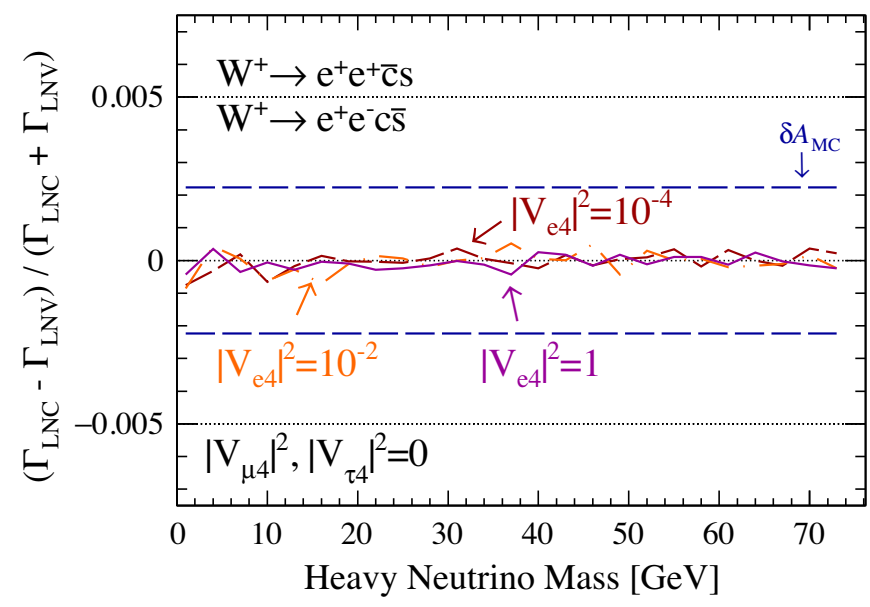

(a)

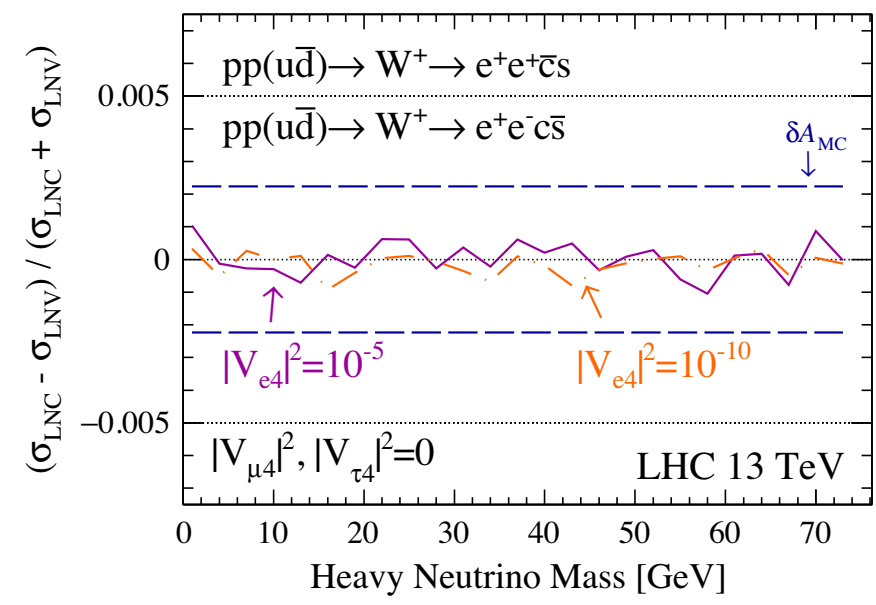

(b)

FIG. 3. (a) Decay rate asymmetry between the $L$-conserving $W^{+} \rightarrow e_{1}^{+} N \rightarrow e_{1}^{+} e_{2}^{-} c \bar{s}\left(\Gamma_{\mathrm{LNC}}\right)$ and $L$-violating $W^{+} \rightarrow e_{1}^{+} N \rightarrow e_{1}^{+} e_{2}^{+} \bar{c} s$ $\left(\Gamma_{\mathrm{LNV}}\right)$ processes as a function of heavy neutrino mass $m_{N}[\mathrm{GeV}]$ for representative active-sterile neutrino mixing $\left|V_{e N}\right|$. (b) Same, but for the hadron-level, cross section asymmetry between the $L$-conserving $p p(u \bar{d}) \rightarrow e_{1}^{+} N \rightarrow e_{1}^{+} e_{2}^{-} c \bar{s}\left(\sigma_{\mathrm{LNC}}\right)$ and $L$-violating $p p(u \bar{d}) \rightarrow$ $e_{1}^{+} e_{2}^{+} \bar{c} s\left(\sigma_{\mathrm{LNV}}\right)$ processes at $\sqrt{s}=13 \mathrm{TeV}$. Also shown are statistical MC uncertainty bands $\left(\delta A_{\mathrm{MC}}\right)$.

above and below zero, reaching at most $\left|\mathcal{A}_{\Gamma}\right| \sim$ $\mathcal{O}\left(1 \times 10^{-3}\right)$, and are consistent with random, statistical noise. ${ }^{4}$ We find that the same behavior holds for all representative choices of active-sterile mixing. This mixing-independent behavior follows from the definition of $\mathcal{A}_{\Gamma}$ in Eq. (5.15), which indicates that normalization factors of $\left|V_{e N}\right|^{2}$ in $\Gamma_{\mathrm{LNC}}$ and $\Gamma_{\mathrm{LNV}}$ cancel in the asymmetry measure. While the absence of a significant asymmetry seems to suggest a total absence of helicity suppression between $\Delta L=0$ and $|\Delta L|=2$ decays of $W$ bosons, we stress that the above processes are dominated by regions of phase space where a heavy neutrino is on or is nearly on its mass shell. As discussed in Sec. IV C, a different behavior is anticipated outside this limit.

\section{Total cross section asymmetry}

As our second measure of helicity suppression in LHC observables, we consider the generalization of the $W$ boson decay chains in Eqs. (5.11)-(5.12). In particular, we consider the $2 \rightarrow 4$-body scattering processes,

$$
\begin{gathered}
\Delta L=0: u \bar{d} \rightarrow W^{+} \rightarrow e_{1}^{+} N \rightarrow e_{1}^{+} e_{2}^{-} c \bar{s}, \\
|\Delta L|=2: u \bar{d} \rightarrow W^{+} \rightarrow e_{1}^{+} N \rightarrow e_{1}^{+} e_{2}^{+} \bar{c} s .
\end{gathered}
$$

We again fix external particle flavors for definiteness and to also avoid interference with the $W W$ scattering process. Diagrams involving $\gamma^{*} / Z^{*}$ exchange are removed in a gauge-invariant manner, while interfering diagrams from

\footnotetext{
${ }^{4}$ While we use the uncertainty estimator $\delta \mathcal{O} / \mathcal{O}=\delta N /$ $N=1 / \sqrt{N}$, for $\mathcal{O}=\Gamma, \sigma$, it is actually an upper limit on the $\mathrm{MC}$ uncertainty due to the sampling and reweighting routines in MGAMC [53].
}

identical particle exchange are kept. As discussed in Sec. IV D, the utility of these processes is that they capture polarization and virtuality effects present in real LHC collisions but not in the idealized decays of Sec. V C.

In analogy to $\mathcal{A}_{\Gamma}$, we use the scattering processes above to build an asymmetry $\mathcal{A}_{\sigma}$ that would arise if helicity suppression were to exist. Specifically, we consider

$$
\begin{aligned}
\mathcal{A}_{\sigma} & \equiv \frac{\sigma\left(u \bar{d} \rightarrow e^{+} e^{-} c \bar{s}\right)-\sigma\left(u \bar{d} \rightarrow e^{+} e^{+} \bar{c} s\right)}{\sigma\left(u \bar{d} \rightarrow e^{+} e^{-} c \bar{s}\right)+\sigma\left(u \bar{d} \rightarrow e^{+} e^{+} \bar{c} s\right)} \\
& \equiv \frac{\sigma_{\mathrm{LNC}}-\sigma_{\mathrm{LNV}}}{\sigma_{\mathrm{LNC}}+\sigma_{\mathrm{LNV}}} .
\end{aligned}
$$

Here we abuse slightly the conventional notation for hadronic cross sections $\sigma(p p \rightarrow \mathcal{B})$ and write explicitly,

$$
\sigma(u \bar{d} \rightarrow \mathcal{B})=f_{u / p} \otimes f_{\bar{d} / p} \otimes \hat{\sigma}(u \bar{d} \rightarrow \mathcal{B}),
$$

to denote that we consider only the $u \bar{d}$ partonic contribution to $p p$ scattering, with $f_{i / p}$ representing the PDF for parton $i$ in hadron $p$, and $\hat{\sigma}$ as the parton-level scattering rate. This is given by the standard expression,

$$
\hat{\sigma}(i j \rightarrow \mathcal{B})=\frac{1}{2 Q^{2} \mathcal{S}_{i} \mathcal{S}_{j} N_{c}^{i} N_{c}^{j}} \int d P S_{\mathcal{B}} \sum_{\text {dof }}|\mathcal{M}(i j \rightarrow \mathcal{B})|^{2} .
$$

To avoid potential washout from beam symmetrization, we do not consider the $\bar{d} u$ partonic channel.

In Fig. 3(b) we show the cross section asymmetry $\mathcal{A}_{\sigma}$ between the $L$-conserving and $L$-violating scattering processes in Eqs. (5.16)-(5.17), as a function of $m_{N}[\mathrm{GeV}]$ for representative active-sterile neutrino mixing $\left|V_{e N}\right|^{2}=10^{-6}$ (solid) and $10^{-10}$ (dash-dot). Also shown is the associated 


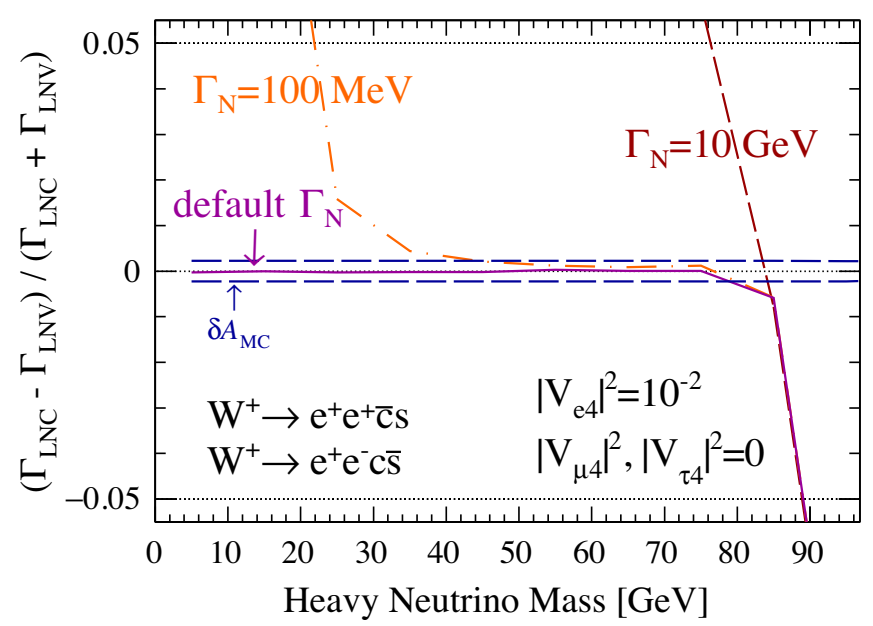

(a)

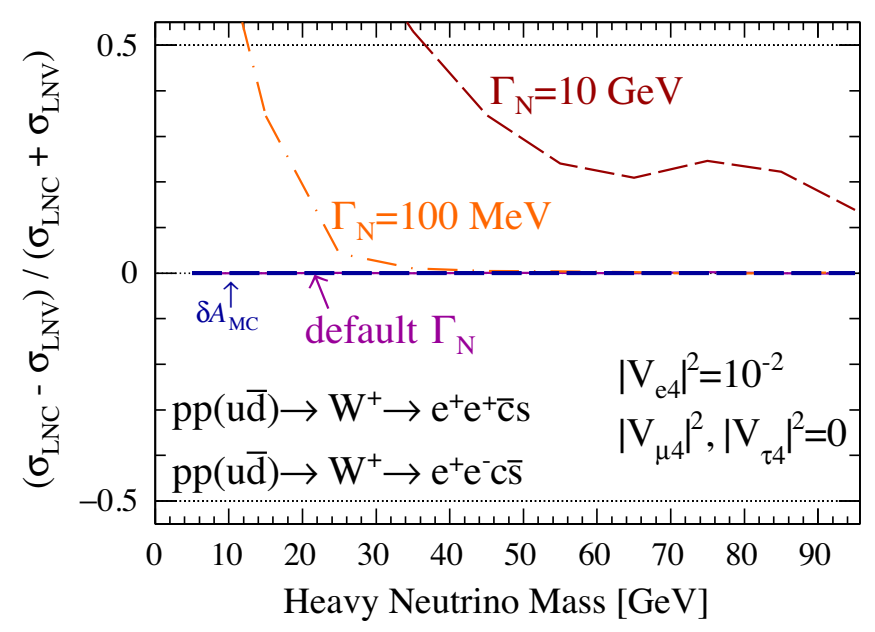

(c)

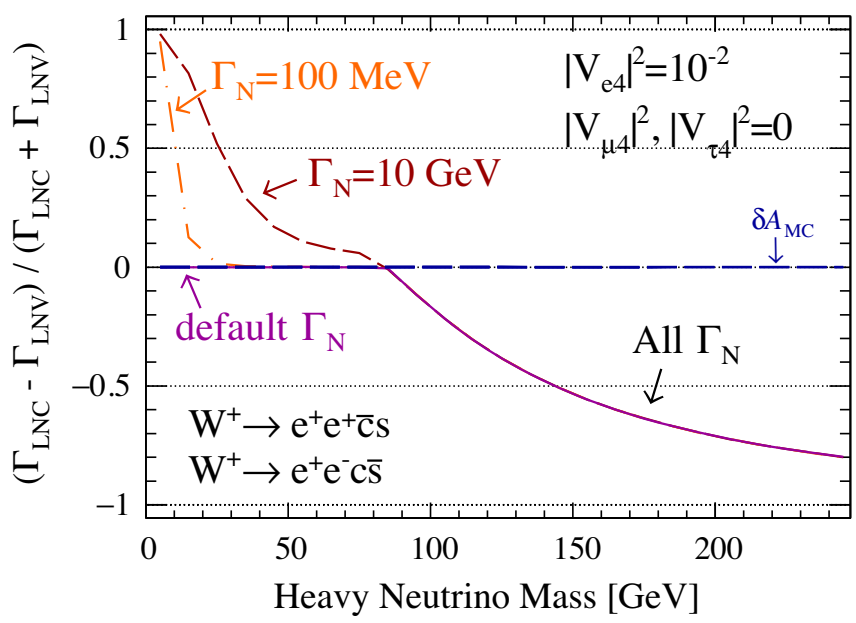

(b)

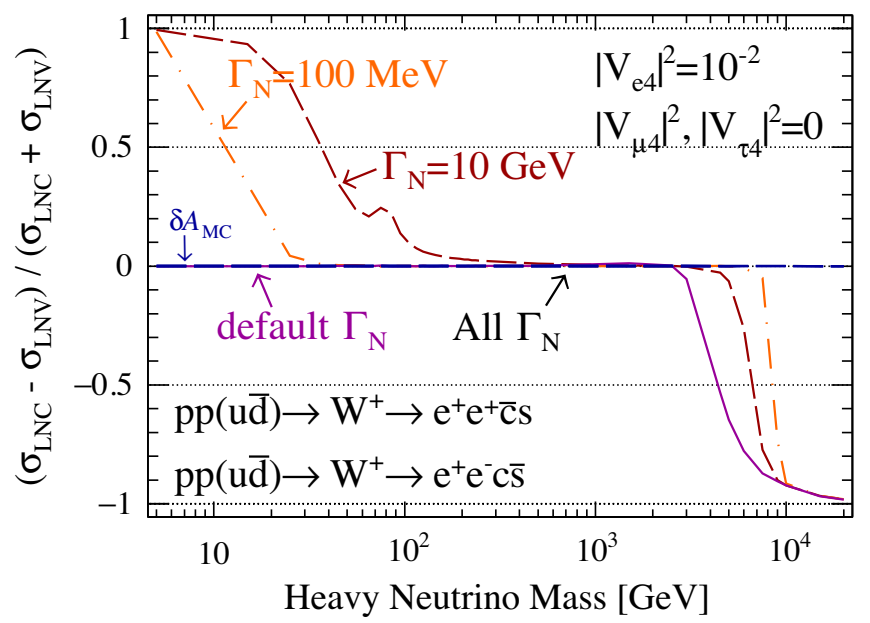

(d)

FIG. 4. (a) Same as Fig. 3(a) but for a total width $\Gamma_{N}$ that varies according to the Lagrangian given in Sec. II (default) as well as at fixed widths of $\Gamma_{N}=100 \mathrm{MeV}$ and $10 \mathrm{GeV}$. For all computations, $\left|V_{e 4}\right|^{2}=10^{-2}$ is assumed with $\left|V_{\mu 4}\right|^{2},\left|V_{\tau 4}\right|^{2}=0$. (b) Same as (a) but for an extended mass range. (c,d) Same as Fig. 3(b) but for the assumptions of (a,b) respectively.

statistical MC uncertainty band $\left(\delta \mathcal{A}_{\mathrm{MC}}\right)$. Remarkably, for heavy neutrino masses in the range of $m_{N} \in[1 \mathrm{GeV}, 75 \mathrm{GeV}]$ we report asymmetries that are statistically consistent with $\mathcal{A}_{\sigma}=0$, i.e., no asymmetry and hence. We find that the same behavior holds for both representative choices of active-sterile mixing.

\section{E. Off-shell effects}

As a final comment, we consider briefly the role of far off-shell effects in $\Delta L=0$ and $|\Delta L|=2$ decays and cross sections through on- and off-shell $W$ bosons. As cautioned above, the $1 \rightarrow 4$ and $2 \rightarrow 4$ transitions that we have so far investigate are dominated by regions of phase space where the intermediate neutrino is (nearly) on-shell. However, as discussed analytically in Sec. IV C and Sec. IV D, vastly different behavior is possible outside this limit. Therefore, to further explore off-shell effects, we consider again the $1 \rightarrow$ 4-body decays in Eqs. (5.11) and (5.12) along with the decay asymmetry measure $\mathcal{A}_{\Gamma}$ in Eq. (5.15). We also consider the $2 \rightarrow$ 4-body scattering processes in Eqs. (5.16) and (5.17) along with the cross section asymmetry measure $\mathcal{A}_{\sigma}$ in Eq. (5.19).

In Fig. 4(a) we plot the width asymmetry for heavy neutrino masses in the range of $m_{N} \in[1 \mathrm{GeV}, 95 \mathrm{GeV}]$ and in Fig. 4(b) for the larger range $m_{N} \in[1 \mathrm{GeV}$, $250 \mathrm{GeV}]$. While we set the relevant active-sterile mixing to be $\left|V_{e 4}\right|^{2}=10^{-2}$ with $\left|V_{\mu 4}\right|^{2},\left|V_{\tau 4}\right|^{2}=0$, we fix $N$ 's total width to be $\Gamma_{N}=100 \mathrm{MeV}$ (dash-dot) and $100 \mathrm{GeV}$ (dash). As a reference we consider as well the width according the Lagrangi an in Sec. II (default, solid). The statical MC uncertainty band is also shown.

For neutrino masses below $M_{W}$ and for both $\Gamma_{N}=$ $100 \mathrm{MeV}$ and $10 \mathrm{GeV}$, we observe the presence of large, positive asymmetries. These indicate the enhancement of the $\Delta L=0$ decay mode over the $|\Delta L|=2$ mode, and in comparison to the benchmark case can be attributed to large-width effects. To understand this in terms of 
kinematics, note that in the absence of a resonance pole the natural scale for $N$ 's virtuality is $p_{N}^{2} \sim M_{W}^{2} \gtrsim m_{N}^{2}$. The enlarged widths allow $N$ 's virtuality to vary away from $p_{N}^{2} \sim m_{N}^{2}$, which then drives a positive asymmetry according to the analytic expression of Eq. (4.43).

We find that $\mathcal{A}_{\Gamma}$ approaches unity for both large-width cases when $m_{N} \lesssim 10 \mathrm{GeV}$, and reduces to about $\mathcal{A}_{\Gamma} \sim 0.5$, for $\Gamma_{N}=100 \mathrm{MeV}(10 \mathrm{GeV})$ when $m_{N} \sim 10(25) \mathrm{GeV}$. For masses above $m_{N} \sim 45 \mathrm{GeV}$ but still below $M_{W}$, the widthto-mass ratio for $\Gamma_{N}=100 \mathrm{MeV}$ is small enough that offshell effects become negligible and the asymmetry vanishes. For $\Gamma_{N}=10 \mathrm{GeV}$ and for all values of $m_{N}<M_{W}$, the asymmetry is always greater than the MC uncertainty band, implying that off-shell effects are always significant. At around $m_{N} \sim 75 \mathrm{GeV}$, i.e., just below the $W$ boson's mass threshold, the asymmetries for the benchmark and $\Gamma_{N}=$ $100 \mathrm{GeV}$ curves begin to move from $\mathcal{A}_{\Gamma} \sim 0$ to subzero values.

For neutrino masses above $M_{W}$ and for all three width cases, we observe the presence of negative asymmetries that slowly approach $\mathcal{A}_{\Gamma}=-1$ for increasing $m_{N}$. This implies a suppression of the $\Delta L=0$ decay mode over the $|\Delta L|=2$ mode, and can be attributed to the faster decoupling of ultra heavy $N$ in the $\Delta L=0$ ME. [For related details, see the discussion following Eq. (4.49).] At values of $m_{N}$ just above $M_{W}$ we find that the width-to-mass ratios for all three cases are negligible enough that all three curves converge. This essentially affirms an insensitivity to the total width in the high-mass limit.

Turning to potential impact of off-shell effects in $2 \rightarrow 4$ scattering, we plot in Figs. 4(c) and 4(d), respectively, the cross section asymmetry measure for heavy neutrino masses in the range of $m_{N} \in[1 \mathrm{GeV}, 95 \mathrm{GeV}]$ and $m_{N} \in[1 \mathrm{GeV}, 250 \mathrm{GeV}]$. We assume the same inputs as in Figs. 4(a) and 4(b) for the decay asymmetry.

For lighter states with $m_{N}<M_{W}$ we observe that the default and $\Gamma_{N}=100 \mathrm{MeV}$ cases exhibit similar qualitative and quantitative behavior as for the decay asymmetry. In these two cases the total widths for $N$ and $W$ are small and satisfy the relation $\Gamma_{N} \ll \Gamma_{W} \ll m_{N} \lesssim M_{W}$. The virtuality carried by the $W$ is therefore comparable to its mass, i.e., $Q \sim M_{W}$, resulting in similar kinematics as in $W$ boson decays. Arguably, for such small $\Gamma_{N}$, one can treat the decays of $W$ bosons with the spin-correlated NWA. When $\Gamma_{N}=10 \mathrm{GeV}$ we observe a cross section asymmetry that is positive and larger than in the decay case. Quantitatively, the asymmetry reaches approximately $\mathcal{A}_{\sigma} \sim 0.75(0.5)$ when $m_{N} \sim 25(35) \mathrm{GeV}$, never drops below $\mathcal{A}_{\sigma} \sim 0.15-0.2$ for $m_{N}<M_{W}$, and briefly grows in the vicinity of $m_{N} \sim M_{W}$. The significant differences between the cross section and decay asymmetries at large $N$ widths, particularly when $\Gamma_{N} \lesssim m_{N} \lesssim M_{W}$, demonstrates a breakdown of the NWA.

When Majorana neutrinos are heavier but still kinematically accessible, i.e., when $M_{W}<m_{N} \ll \sqrt{s}$, the cross section asymmetry rapidly shrinks for each choice of $\Gamma_{N}$.
More specifically, the asymmetry drops to $\mathcal{A}_{\sigma} \sim 0.01$ when $m_{N} \sim 35(500) \mathrm{GeV}$ and $\Gamma_{N}=100 \mathrm{MeV}(10 \mathrm{GeV})$, and continues toward zero for larger $m_{N} .\left|\mathcal{A}_{\sigma}\right|$ remains small, if not negligible, until $m_{N} \sim 2-5 \mathrm{TeV}$. At these masses the cross section asymmetries migrate to negative values of $\mathcal{A}_{\sigma}$, again indicating a $L$-violating cross section that is larger than the analogous $L$-conserving rate. We observe a hierarchy in this behavior, with larger $\Gamma_{N}$ leading to more negative values of $\mathcal{A}_{\sigma}$. (Note that due to the opening of new decay modes, $\Gamma_{N}^{\text {default }} \gg 10 \mathrm{GeV}$ at such large values of $m_{N}$.)

When $N$ is no long kinematically accessible, i.e., when $M_{W} \ll \sqrt{s} \lesssim m_{N}$, one again enters the decoupling phase. Here we observe observe similar behavior as found in the $m_{N}>M_{W}$ regime of the decay asymmetry in Fig. 4(b). At these scales, the total widths of $N$ are irrelevant as it is always far off-shell. As such, all three curves converge at around $m_{N} \sim 10 \mathrm{TeV}$ and tend toward $\mathcal{A}_{\sigma}=-1$ for increasing heavy neutrino masses. Using again the language of effective field theories, the negative-valued cross section asymmetry follows from the $L$-violating process occurring at dimension seven, whereas the $L$-conserving process occurs at dimension eight. The latter rate is thus relatively suppressed by a factor of $\left(Q / m_{N}\right)^{2} \ll 1$.

\section{SUMMARY AND CONCLUSIONS}

Whether or not light neutrinos are Majorana fermions remains one of the most pressing open questions in particle physics today. If neutrinos are their own antiparticle, then it is likely that new particles and interactions play a role in generating neutrino masses that are hierarchically smaller than the EW scale. Hence, establishing the Majorana nature of neutrinos is a stepping stone to more fully understanding the fundamental symmetries of nature.

In this study, we report an analytical and numerical investigation into the impact of helicity inversion on partial widths and cross sections of $|\Delta L|=2$ processes at the LHC. We focus as a case study on $L$-conserving and $L$-violating, 4-body decays of $W$ bosons mediated by a heavy Majorana neutrino $N$ in the phenomenological type I seesaw model. After isolating the relative helicity preservation (inversion) in the $L$-conserving (violating) process at the ME level in Sec. IV A (IV B), we show that up to finite-width effects an identical dependence on $N$ 's mass $\left(m_{N}\right)$ emerges at the squared ME level due to the different scaling of 4-momenta and squared 4-momenta. When $N$ goes on-shell, we find that this mass dependence precisely cancels. This renders total decay and scattering rates equal and nonzero, even for small $m_{N}$. We go on to find in Sec. IV C that for far off-shell $N$, large differences between the $\Delta L=0$ and $|\Delta L|=2$ squared MEs can arise. Depending on the precise off-shell limit, this can lead to a relative enhancement or suppression of the $L$-violating channel. In Sec. IV D, we show that our findings extend to $2 \rightarrow 4$ scattering, and in Sec. IV E to other neutrino mass models, so long as consistent propagation of helicity inversion is taken into account. 
In Sec. V we quantify our analytic results by performing exact numerical ME computations using the MC event generator MadGraph5_aMC@NLO in conjunction with the HeavyN model libraries. Starting in Sec. V B, we confirm the strong presence of helicity inversion in the $W \rightarrow N e$ decay process using our MC setup. We then move onto the more general $1 \rightarrow 4$ decay and $2 \rightarrow 4$ scattering processes in Secs. V C and VD, respectively. After building asymmetries $(\mathcal{A})$ sensitive to helicity suppression and enhancements in $L$-violating processes, we report the absence of numerically significant helicity suppression despite the presence of helicity inversion. In Sec. VE we find that far off-shell contributions can lead to numerically significant helicity enhancement or suppression of $|\Delta L|=2$ channels, particularly in the decoupling limit, as well as to a breakdown of the NWA. In all cases, we find strong agreement with analytical expectations for on- and off-shell Majorana neutrinos.

Taking everything together, we condense our findings into the following model-dependent conclusions:

(i) When a Majorana neutrino $N$ can be on-shell and its width $\Gamma_{N}$ is small, i.e., $\Gamma_{N} \ll m_{N}$, then $L$-conserving and $L$-violating rates are the same.

(ii) When $N$ can be on-shell but its width is large, i.e., $\Gamma_{N} \sim m_{N}$, then off-shell/finite-width effects trigger $L$-conserving rates larger than $L$-violating rates.

(iii) When $N$ is too heavy to be on-shell, e.g., $m_{N} \gtrsim \sqrt{s}$, then off-shell/decoupling effects trigger $L$-violating rates that are larger than $L$-conserving rates.
We stress that more could be learned by further investigating finite width effects as well as the criteria for applying the NWA when Majorana fermions are present. More can also be learned about the potential loop-level generation of helicity asymmetries $\mathcal{A}$ as well as the role of additional new particles with chiral interactions. We strongly encourage future studies.

\section{ACKNOWLEDGMENTS}

Didar Dobur and Lesya Shchutska are acknowledge for discussions that initiated this work. André de Gouvêa and Boris Kayser are thanked for their comments on an early version of this manuscript. Carlos Alishaan, Alexey Boyarsky, Albert De Roeck, and Olivier Mattelaer are also thanked for their helpful input. This work has received funding from the European Union's Horizon 2020 research and innovation programme as part of the Marie Skłodowska-Curie Innovative Training Network MCnetITN3 (Grant Agreement No. 722104), FNRS "Excellence of Science" EOS be.h Project No. 30820817. This work is also supported under the UCLouvain fund "MOVE-IN Louvain." Computational resources have been provided by the supercomputing facilities of the Universite catholique de Louvain (CISM/ UCL) and the Consortium des Equipements de Calcul Intensif en Fédération Wallonie Bruxelles (CÉCI) funded by the Fond de la Recherche Scientifique de Belgique (F. R. S.-FNRS) under convention 2.5020 .11 and by the Walloon Region.
[1] European Strategy Group, https://doi.org/10.17181/ ESU2020.

[2] E. Ma, Phys. Rev. Lett. 81, 1171 (1998).

[3] P. Minkowski, Phys. Lett. 67B, 421 (1977).

[4] W. Konetschny and W. Kummer, Phys. Lett. 70B, 433 (1977).

[5] T. Yanagida, Conf. Proc. C 7902131, 95 (1979), https:// inspirehep.net/literature/143150.

[6] S. L. Glashow, NATO Sci. Ser. B 61, 687 (1980), https:// inspirehep.net/literature/144466.

[7] R. N. Mohapatra and G. Senjanovic, Phys. Rev. Lett. 44, 912 (1980).

[8] M. Gell-Mann, P. Ramond, and R. Slansky, Conf. Proc. C 790927, 315 (1979), https://inspirehep.net/literature/9686.

[9] R. E. Shrock, Phys. Rev. D 24, 1232 (1981).

[10] J. Schechter and J. W. F. Valle, Phys. Rev. D 22, 2227 (1980).

[11] T. P. Cheng and L. F. Li, Phys. Rev. D 22, 2860 (1980).

[12] G. Lazarides, Q. Shafi, and C. Wetterich, Nucl. Phys. B181, 287 (1981).

[13] R. N. Mohapatra and G. Senjanovic, Phys. Rev. D 23, 165 (1981).
[14] A. Zee, Phys. Lett. 93B, 389 (1980); 95B, 461(E) (1980).

[15] L. J. Hall and M. Suzuki, Nucl. Phys. B231, 419 (1984).

[16] R. Foot, H. Lew, X. G. He, and G. C. Joshi, Z. Phys. C 44, 441 (1989).

[17] J. C. Pati and A. Salam, Phys. Rev. D 10, 275 (1974); 11, 703(E) (1975).

[18] R. N. Mohapatra and J. C. Pati, Phys. Rev. D 11, 566 (1975).

[19] R. N. Mohapatra and J. C. Pati, Phys. Rev. D 11, 2558 (1975).

[20] G. Senjanovic and R. N. Mohapatra, Phys. Rev. D 12, 1502 (1975).

[21] P. Langacker, Phys. Rep. 72, 185 (1981).

[22] B. Bajc and G. Senjanovic, J. High Energy Phys. 08 (2007) 014.

[23] B. Kayser, Phys. Rev. D 26, 1662 (1982).

[24] R. N. Mohapatra and J. C. Pati, Phys. Rev. D 11, 566 (1975).

[25] A. Atre, T. Han, S. Pascoli, and B. Zhang, J. High Energy Phys. 05 (2009) 030.

[26] V. Tello, M. Nemevsek, F. Nesti, G. Senjanovic, and F. Vissani, Phys. Rev. Lett. 106, 151801 (2011).

[27] F. F. Deppisch, P. S. Bhupal Dev, and A. Pilaftsis, New J. Phys. 17, 075019 (2015). 
[28] Y. Cai, T. Han, T. Li, and R. Ruiz, Front. Phys. 6, 40 (2018).

[29] V. Cirigliano, W. Dekens, J. de Vries, M. L. Graesser, and E. Mereghetti, J. High Energy Phys. 12 (2018) 097.

[30] M. J. Dolinski, A. W. P. Poon, and W. Rodejohann, Annu. Rev. Nucl. Part. Sci. 69, 219 (2019).

[31] A. Pilaftsis, Z. Phys. C 55, 275 (1992).

[32] J. Kersten and A. Y. Smirnov, Phys. Rev. D 76, 073005 (2007).

[33] S. Antusch and O. Fischer, J. High Energy Phys. 05 (2015) 053.

[34] K. Moffat, S. Pascoli, and C. Weiland, arXiv:1712.07611.

[35] T. Han, I. Lewis, R. Ruiz, and Z. g. Si, Phys. Rev. D 87, 035011 (2013); 87, 039906(E) (2013).

[36] C. Y. Chen, P. S. B. Dev, and R. N. Mohapatra, Phys. Rev. D 88, 033014 (2013).

[37] J. Gluza, T. Jelinski, and R. Szafron, Phys. Rev. D 93, 113017 (2016).

[38] R. Ruiz, Eur. Phys. J. C 77, 375 (2017).

[39] C. Arbelaéz, C. Dib, I. Schmidt, and J. C. Vasquez, Phys. Rev. D 97, 055011 (2018).

[40] A. Baha Balantekin and B. Kayser, Annu. Rev. Nucl. Part. Sci. 68, 313 (2018).

[41] A. B. Balantekin, A. de Gouvêa, and B. Kayser, Phys. Lett. B 789, 488 (2019).

[42] P. Hernández, J. Jones-Pérez, and O. Suarez-Navarro, Eur. Phys. J. C 79, 220 (2019).

[43] T. Fukuyama, S. Kanda, D. Nomura, and K. Shimomura, arXiv:1908.01630.

[44] W. Y. Keung and G. Senjanovic, Phys. Rev. Lett. 50, 1427 (1983).

[45] A. Ferrari, J. Collot, M. L. Andrieux, B. Belhorma, P. de Saintignon, J. Y. Hostachy, P. Martin, and M. Wielers, Phys. Rev. D 62, 013001 (2000).

[46] D. Berdine, N. Kauer, and D. Rainwater, Phys. Rev. Lett. 99, 111601 (2007).

[47] N. Kauer, Phys. Lett. B 649, 413 (2007).

[48] N. Kauer, J. High Energy Phys. 04 (2008) 055.

[49] C. F. Uhlemann and N. Kauer, Nucl. Phys. B814, 195 (2009).

[50] P. Artoisenet, R. Frederix, O. Mattelaer, and R. Rietkerk, J. High Energy Phys. 03 (2013) 015.

[51] D. Binosi, J. Collins, C. Kaufhold, and L. Theussl, Comput. Phys. Commun. 180, 1709 (2009).

[52] T. Stelzer and W. F. Long, Comput. Phys. Commun. 81, 357 (1994).

[53] J. Alwall, R. Frederix, S. Frixione, V. Hirschi, F. Maltoni, O. Mattelaer, H. S. Shao, T. Stelzer, P. Torrielli, and M. Zaro, J. High Energy Phys. 07 (2014) 079.

[54] D. Alva, T. Han, and R. Ruiz, J. High Energy Phys. 02 (2015) 072.

[55] C. Degrande, O. Mattelaer, R. Ruiz, and J. Turner, Phys. Rev. D 94, 053002 (2016).

[56] F. del Aguila and J. A. Aguilar-Saavedra, Nucl. Phys. B813, 22 (2009).

[57] S. Pascoli, R. Ruiz, and C. Weiland, J. High Energy Phys. 06 (2019) 049.

[58] T. Asaka and M. Shaposhnikov, Phys. Lett. B 620, 17 (2005).

[59] T. Asaka, S. Blanchet, and M. Shaposhnikov, Phys. Lett. B 631, 151 (2005).
[60] R. N. Mohapatra, Phys. Rev. Lett. 56, 561 (1986).

[61] R. N. Mohapatra and J. W. F. Valle, Phys. Rev. D 34, 1642 (1986).

[62] J. Bernabeu, A. Santamaria, J. Vidal, A. Mendez, and J. W. F. Valle, Phys. Lett. B 187, 303 (1987).

[63] M. Blennow, P. Coloma, E. Fernandez-Martinez, J. Hernandez-Garcia, and J. Lopez-Pavon, J. High Energy Phys. 04 (2017) 153.

[64] E. Fernandez-Martinez, J. Hernandez-Garcia, and J. LopezPavon, J. High Energy Phys. 08 (2016) 033.

[65] M. Dentler, Á. Hernández-Cabezudo, J. Kopp, P. A. N. Machado, M. Maltoni, I. Martinez-Soler, and T. Schwetz, J. High Energy Phys. 08 (2018) 010.

[66] I. Esteban, M. C. Gonzalez-Garcia, M. Maltoni, T. Schwetz, and A. Zhou, J. High Energy Phys. 09 (2020) 178.

[67] F. Maltoni and T. Stelzer, J. High Energy Phys. 02 (2003) 027.

[68] P. de Aquino, W. Link, F. Maltoni, O. Mattelaer, and T. Stelzer, Comput. Phys. Commun. 183, 2254 (2012).

[69] V. Hirschi, R. Frederix, S. Frixione, M. V. Garzelli, F. Maltoni, and R. Pittau, J. High Energy Phys. 05 (2011) 044.

[70] J. Alwall, C. Duhr, B. Fuks, O. Mattelaer, D. G. Öztürk, and C. H. Shen, Comput. Phys. Commun. 197, 312 (2015).

[71] K. Hagiwara and D. Zeppenfeld, Nucl. Phys. B313, 560 (1989).

[72] K. Hagiwara and D. Zeppenfeld, Nucl. Phys. B274, 1 (1986).

[73] K. Hagiwara, R. D. Peccei, D. Zeppenfeld, and K. Hikasa, Nucl. Phys. B282, 253 (1987).

[74] K. Hagiwara and D. Zeppenfeld, Nucl. Phys. B313, 560 (1989).

[75] J. Alwall, M. Herquet, F. Maltoni, O. Mattelaer, and T. Stelzer, J. High Energy Phys. 06 (2011) 128.

[76] N. D. Christensen and C. Duhr, Comput. Phys. Commun. 180, 1614 (2009).

[77] C. Degrande, C. Duhr, B. Fuks, D. Grellscheid, O. Mattelaer, and T. Reiter, Comput. Phys. Commun. 183, 1201 (2012).

[78] A. Alloul, N. D. Christensen, C. Degrande, C. Duhr, and B. Fuks, Comput. Phys. Commun. 185, 2250 (2014).

[79] A. Denner, H. Eck, O. Hahn, and J. Kublbeck, Nucl. Phys. B387, 467 (1992).

[80] A. Denner, H. Eck, O. Hahn, and J. Kublbeck, Phys. Lett. B 291, 278 (1992).

[81] D. Buarque Franzosi, O. Mattelaer, R. Ruiz, and S. Shil, J. High Energy Phys. 04 (2020) 082.

[82] A. D. Martin, W. J. Stirling, R. S. Thorne, and G. Watt, Eur. Phys. J. C 63, 189 (2009).

[83] A. Buckley, J. Ferrando, S. Lloyd, K. Nordström, B. Page, M. Rüfenacht, M. Schönherr, and G. Watt, Eur. Phys. J. C 75, 132 (2015).

[84] T. Appelquist and J. Carazzone, Phys. Rev. D 11, 2856 (1975).

[85] R. K. Ellis, W. J. Stirling, and B. R. Webber, $Q C D$ and Collider Physics (Cambridge University Press, Cambridge, England, 2011), https://inspirehep.net/literature/328604.

[86] Z. Bern, G. Diana, L. J. Dixon, F. Febres Cordero, D. Forde, T. Gleisberg, S. Hoeche, H. Ita, D. A. Kosower, and D. Maitre et al., Phys. Rev. D 84, 034008 (2011). 
[87] W. J. Stirling and E. Vryonidou, J. High Energy Phys. 07 (2012) 124.

[88] M. Nemevšek, F. Nesti, and G. Popara, Phys. Rev. D 97, 115018 (2018).

[89] S. Bar-Shalom, N. G. Deshpande, G. Eilam, J. Jiang, and A. Soni, Phys. Lett. B 643, 342 (2006).

[90] Z. Si and K. Wang, Phys. Rev. D 79, 014034 (2009).

[91] N. Liu, Z. G. Si, L. Wu, H. Zhou, and B. Zhu, Phys. Rev. D 101, 071701 (2020).
[92] R. Ruiz, J. High Energy Phys. 12 (2015) 165.

[93] N. Arkani-Hamed, S. Dimopoulos, G. R. Dvali, and J. March-Russell, Phys. Rev. D 65, 024032 (2001).

[94] Y. Grossman and M. Neubert, Phys. Lett. B 474, 361 (2000).

[95] C. Csaki, C. Delaunay, C. Grojean, and Y. Grossman, J. High Energy Phys. 10 (2008) 055.

[96] P. Coloma, E. Fernández-Martínez, M. González-López, J. Hernández-García, and Z. Pavlovic, arXiv:2007.03701. 